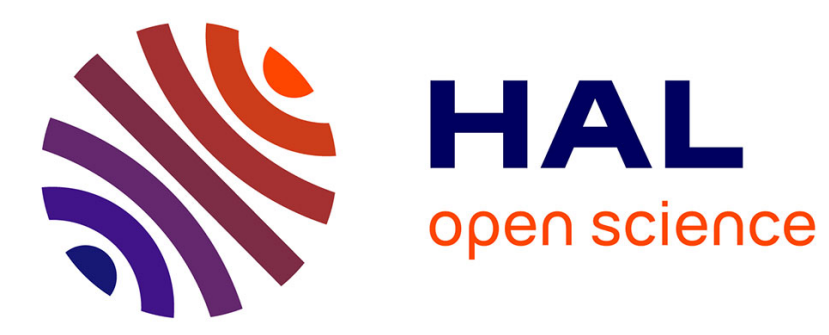

\title{
Change Detection in Multilook Polarimetric SAR Imagery With Determinant Ratio Test Statistic
}

\author{
N. Bouhlel, V. Akbari, S. Meric
}

\section{To cite this version:}

N. Bouhlel, V. Akbari, S. Meric. Change Detection in Multilook Polarimetric SAR Imagery With Determinant Ratio Test Statistic. IEEE Transactions on Geoscience and Remote Sensing, 2020, pp.115. 10.1109/TGRS.2020.3043517 . hal-03164088

\section{HAL Id: hal-03164088 \\ https://hal.science/hal-03164088}

Submitted on 31 Mar 2021

HAL is a multi-disciplinary open access archive for the deposit and dissemination of scientific research documents, whether they are published or not. The documents may come from teaching and research institutions in France or abroad, or from public or private research centers.
L'archive ouverte pluridisciplinaire HAL, est destinée au dépôt et à la diffusion de documents scientifiques de niveau recherche, publiés ou non, émanant des établissements d'enseignement et de recherche français ou étrangers, des laboratoires publics ou privés. 


\title{
Change Detection in Multilook Polarimetric SAR Imagery With Determinant Ratio Test Statistic
}

\author{
Nizar Bouhlel $^{\circledR}$, Member, IEEE, Vahid Akbari ${ }^{\circledR}$, Member, IEEE, and Stéphane Méric ${ }^{\circledR}$, Member, IEEE
}

\begin{abstract}
In this article, we propose a determinant ratio test (DRT) statistic to measure the similarity of two covariance matrices for unsupervised change detection in polarimetric radar images. The multilook complex covariance matrix is assumed to follow a scaled complex Wishart distribution. In doing so, we provide the distribution of the DRT statistic that is exactly Wilks's lambda of the second kind distribution, with density expressed in terms of Meijer G-functions. Due to this distribution, the constant false alarm rate (CFAR) algorithm is derived in order to achieve the required performance. More specifically, a threshold is provided by the CFAR to apply to the DRT statistic producing a binary change map. Finally, simulated and real multilook polarimetric SAR (PolSAR) data are employed to assess the performance of the method and is compared with the Hotelling-Lawley trace (HLT) statistic and the likelihood ratio test (LRT) statistic.
\end{abstract}

Index Terms-Change detection, complex Wishart distribution, determinant ratio test (DRT), Hotelling-Lawley trace (HLT), likelihood ratio test (LRT), multilook polarimetric synthetic aperture radar (SAR) data, Wilks's lambda of the second kind distribution.

\section{INTRODUCTION}

$\mathbf{T}$ HE synthetic aperture radar (SAR) image change detection has become very important in remote sensing for monitoring dynamic processes on the Earth, for instance, damage assessment in urban areas, deforestation and clearcut detection, flooding, and monitoring of glaciers. Change detection is a process that analyzes multitemporal remote sensing images acquired on the same geographical area for identifying changes that occurred at distinct observation dates. The result is a generation of a change detection map in which changed areas are explicitly identified.

Several unsupervised change detection methods have been proposed in the literature. Two families of methods handle the change detection process: the statistical information theory and the hypothesis test theory. The first family is based on

Manuscript received June 1, 2020; revised October 19, 2020; accepted November 29, 2020. (Corresponding author: Nizar Bouhlel.)

Nizar Bouhlel was with the Team Signal Communication and Embedded Electronics (SCEE), Institut d'Electronique et de Telecommunications de Rennes (IETR), CentraleSupélec, 35042 Rennes, France. He is now with the Institut Agro, Statistics and Computer Science Department, 49045 Angers, France (e-mail: nizar.bouhlel@free.fr; nizar.bouhlel@centralesupelec.fr).

Vahid Akbari is with the Department of Computing Science and Mathematics, University of Stirling, Stirling 9037, U.K.

Stéphane Méric is with the Department of Image and Remote Sensing, IETR (UMR CNRS 6164), 35708 Rennes, France.

Color versions of one or more figures in this article are available at https://doi.org/10.1109/TGRS.2020.3043517.

Digital Object Identifier 10.1109/TGRS.2020.3043517 information-theoretic measures in order to design a distance between images. In doing so, surrounding pixels are modeled by a given distribution and distance measures are applied to obtain a comparative statistic. With this family, the change detection is less sensitive to acquisition conditions since the used methodology considers information from the neighborhood. This information is relevant when dealing with data corrupted by speckle or/and the number of looks is low: to name a few of statistical information distances: mutual information [1], [2], variational and mixed information [1], [3], and stochastic distances such as the Kullback-Leibler, Rényi, Bhattacharyya, and Hellinger distances [4].

The second family aims to compute a covariance equality test and to test the hypotheses of change versus no-change where an asymptotic distribution is usually employed by the test statistic. The simplest one is to compute the ratio of SAR amplitudes or intensities observed at different times. This kind of ratios is a well-known test statistic in singlechannel SAR-based change detection [5]. A large number of test statistics have been developed and described in the literature for automatic and unsupervised change detection, such as mean ratio/log-ratio measures [6], [7], Gauss log ratios [8], multitemporal coherence analysis [6], and maximumlikelihood ratios [9].

Polarimetric SAR (PolSAR) gives more scattering information than single-polarization channel SAR data, which can be used to detect the change and increase the quality of the change detection map. The first work on test statistics for change detection in multilook PolSAR data was proposed by Conradsen et al. [10]. They proposed a likelihood ratio test (LRT) for the equality of two complex covariance matrices $\boldsymbol{\Sigma}_{1}$ and $\boldsymbol{\Sigma}_{2}$ and gave the approximated distribution of the LRT statistic. The LRT algorithm worked by comparing two hypotheses: the null hypothesis $\left(H_{0}\right)$ corresponding to "no-change" and an alternative hypothesis $\left(H_{1}\right)$ corresponding to "change." The LRT approach was extended to the multitemporal case [11], [12] and multifrequency data [13]. Kersten et al. [14] compared three test statistics: the LRT statistic also called the Bartlett test, the contrast ratio test, and the ellipticity test. The second one was based on the largest and the smallest eigenvalues of $\Sigma_{1} \Sigma_{2}^{-1}$, and then, the change was represented by these eigenvalues. The third test statistic was based on the combination of the determinant and the trace of $\Sigma_{1} \Sigma_{2}^{-1}$ by using the eigenvalues.

Later, Akbari et al. [5] proposed a simpler test statistic to detect changes in many scenarios. The test assumed the 
scaled complex Wishart distribution for the covariance matrix data. It was based on the complex-kind Hotelling-Lawley trace (HLT) that was used to measure the similarity of two covariance matrices. They applied a decision threshold to the test statistic to detect changes. The threshold was determined using the constant false alarm rate (CFAR) algorithm [15]. Akbari et al. proposed the Fisher-Snedecor (FS) distribution as the approximation of the sampling distribution of the HLT test statistic. The parameters of the FS distribution depended only on the dimension of the polarimetric data and the equivalent number of looks (ENL) estimated for images. They used the method of the matrix log cumulants [16] to estimate the ENL.

In this article, the ratio of the determinant of two matrices is proposed as a new test statistic to measure the similarity of two covariance matrices that are assumed to follow scaled complex Wishart distributions. The new test is called the determinant ratio test (DRT) statistic and it is able to produce a scalar value, to which a threshold is applied. The distribution of the test statistic under the null hypothesis is exactly Wilks's lambda of the second kind distribution with density expressed in terms of Meijer G-functions [17]. Wilks's lambda distribution corresponds to the product of $d$ independent Beta distributed of the second kind random variables, where $d$ is the number of polarimetric channels. The latter distribution depends on the dimension of the polarimetric data and the ENL.

For the production of a binary change detection map, a threshold is applied to the test statistic. Several thresholding methods have been suggested in the literature to determine the threshold in a completely unsupervised manner: to name a few of them, CFAR algorithm [15], Otsu's method [18], and Kittler and Illingworth (K\&I) algorithm [19], [20]. In this article, we limit ourselves to the CFAR algorithm since we are more interested in the test statistic rather than the thresholding method. Consequently, the final binary change map is made at a predefined false alarm rate (FAR). To further illustrate the potential of our method in change detection for multilook PolSAR data, the well-known HLT and LRT test statistics for measuring the equality of two multilook covariance matrices are compared with our approach. Simulated and real multilook PolSAR data are used for this comparison.

This article is organized as follows. Section II introduces the statistical model for the covariance matrix data. Section III describes some properties related to the scaled complex Wishart distribution, the DRT for equality of two complex scaled Wishart distributions, and its sampling distribution. In Section IV, the proposed polarimetric change detection algorithm based on the DRT is presented, followed by the CFAR principle to determine the threshold. The HLT and LRT methods are briefly shown for a later comparison with our method. Section $\mathrm{V}$ demonstrates the performance of the method with a simulated and real PolSAR data set and discusses the results. Section VI is dedicated to a summary and a conclusion.

\section{Multilook PolSaR Image Model}

The polarimetric scattering vector is defined as

$$
\mathbf{s}=\left[s_{\mathrm{hh}}, s_{\mathrm{hv}}, s_{\mathrm{vh}}, s_{\mathrm{vv}}\right]^{T} \in \mathbb{C}^{d}
$$

with $s_{x y}$ representing the complex scattering coefficients, where $x$ is the transmit and $y$ is the receive polarization. Moreover, $h$ denotes horizontal, $v$ denotes vertical [21], [.] $]^{T}$ means transposition, and $d=\operatorname{dim}(\mathbf{s})$ is the vector dimension. The vector $\mathbf{s}$ is a single-look polarimetric complex format representation of PolSAR data. It is assumed that $\mathbf{s}$ is a $d$-dimensional speckle vector, which follows a circular complex Gaussian distribution $\left(\mathbf{s} \sim \mathcal{N}_{d}^{\mathbb{C}}(0, \boldsymbol{\Sigma})\right.$ ), with a zero-mean vector and a covariance matrix $\boldsymbol{\Sigma}$. The multilooking of PolSAR data reduces the speckle effect characteristic of coherent imaging systems. The polarimetric multilooking operation is given by

$$
\mathbf{X}=\frac{1}{L} \sum_{\ell=1}^{L} \mathbf{s}_{\ell} \mathbf{s}_{\ell}^{H}, \quad L \geq d
$$

where $L$ is the number of looks, $(.)^{H}$ denotes the Hermitian operator, and $\mathbf{X} \in \boldsymbol{\Omega}_{+} \subset \mathbb{C}^{d \times d}$ is the multilook polarimetric covariance matrix considered as a random matrix defined on the cone, denoted $\boldsymbol{\Omega}_{+}$, of the positive definite complex Hermitian matrices. When $L \geq d$, the unnormalized sample covariance matrix defined as $\mathbf{Z}=L \mathbf{X}$ follows the nonsingular complex Wishart distribution [22] denoted as $\mathbf{Z} \sim \mathcal{W}_{d}^{\mathbb{C}}(L, \boldsymbol{\Sigma})$ and $\mathbf{X}$ follows a scaled complex Wishart distribution, denoted $\mathbf{X} \sim \operatorname{s\mathcal {W}}_{d}^{\mathbb{C}}(L, \boldsymbol{\Sigma})$, with a probability density function (pdf) given by $f_{\mathbf{X}}(\mathbf{X})=f_{\mathbf{Z}}(L \mathbf{X})\left|J_{\mathbf{Z} \rightarrow \mathbf{X}}\right|$, where $\left|J_{\mathbf{Z} \rightarrow \mathbf{X}}\right|=L^{d^{2}}$ is the Jacobian determinant of the transformation $\mathbf{Z}=L \mathbf{X}$ [23]. The pdf of $\mathbf{X}$ is

$$
f_{\mathbf{X}}(\mathbf{X})=\frac{L^{L d}|\mathbf{X}|^{L-d}}{\Gamma_{d}(L)|\mathbf{\Sigma}|^{L}} \operatorname{etr}\left(-L \boldsymbol{\Sigma}^{-1} \mathbf{X}\right)
$$

where $\operatorname{etr}()=.\exp (\operatorname{tr}()$.$) is the exponential trace operator,$ I.| is the determinant operator, and $\Gamma_{d}(L)$ is the multivariate gamma function of the complex kind defined as

$$
\Gamma_{d}(L)=\pi^{d(d-1) / 2} \prod_{i=0}^{d-1} \Gamma(L-i)
$$

where $\Gamma(L)$ is the standard Euler gamma function.

\section{THEORY}

This section describes some properties related to the scaled complex Wishart distribution and the DRT for equality of two complex scaled Wishart distributions.

Theorem 1 (Goodman [24]): Let $\mathbf{X}$ be a complex Hermitian positive definite random $d \times d$ matrix that follows a scaled complex Wishart distribution, $\mathbf{X} \sim \operatorname{s\mathcal {W}}_{d}^{\mathbb{C}}(L, \boldsymbol{\Sigma})$. The random variable $(2 L)^{d}|\mathbf{X}| /|\boldsymbol{\Sigma}|$ is distributed as the product of $d$ independent chi-square $\left(\chi^{2}\right)$ distributed random variables with $2 L, 2(L-1), \ldots, 2(L-d+1)$ degrees of freedom, respectively, i.e.,

$$
(2 L)^{d} \frac{|\mathbf{X}|}{|\mathbf{\Sigma}|} \sim \prod_{i=0}^{d-1} \chi^{2}(2(L-i)) .
$$

Proof: The characteristic functions of the random variable $\ln \left((2 L)^{d}|\mathbf{X}| /|\boldsymbol{\Sigma}|\right)$ and $\ln \left(\prod_{i=0}^{d-1} \chi^{2}(2(L-i))\right)$ are computed and seen to be equal (for more details, see Appendix A). 
Corollary 2: Let $\mathbf{X}$ and $\mathbf{Y}$ be two complex Hermitian positive definite random $d \times d$ matrices that follow scaled complex Wishart distributions defined as:

$$
\mathbf{X} \sim \operatorname{s\mathcal {W}}_{d}^{\mathbb{C}}\left(L_{x}, \boldsymbol{\Sigma}\right) \text { and } \mathbf{Y} \sim s \mathcal{W}_{d}^{\mathbb{C}}\left(L_{y}, \boldsymbol{\Sigma}\right) .
$$

The random variable defined by the determinant ratio of $L_{x} \mathbf{X}$ and $L_{y} \mathbf{Y}$ is distributed as the product of $d$ independent Beta distributed of the second kind (also called Beta prime) random variables with parameters $\left(L_{x}-i\right)$ and $\left(L_{y}-i\right)$

$$
\frac{\left|L_{x} \mathbf{X}\right|}{\left|L_{y} \mathbf{Y}\right|} \sim \prod_{i=0}^{d-1} \operatorname{Beta}^{\mathrm{II}}\left(L_{x}-i, L_{y}-i\right)
$$

where the pdf for $\operatorname{Beta}^{\mathrm{II}}\left(L_{x}-i, L_{y}-i\right)$ is given by

$$
\begin{aligned}
f_{i}(x) & =\frac{\Gamma\left(L_{x}+L_{y}-2 i\right)}{\Gamma\left(L_{x}-i\right) \Gamma\left(L_{y}-i\right)} \frac{x^{L_{x}-i-1}}{(1+x)^{L_{x}+L_{y}-2 i}} \\
& =\frac{1}{\Gamma\left(L_{x}-i\right) \Gamma\left(L_{y}-i\right)} G_{1,1}^{1,1}\left(\begin{array}{c}
-L_{y}+i \\
L_{x}-i-1
\end{array} \mid x\right)
\end{aligned}
$$

where $G_{1,1}^{1,1}(\cdot \mid$.$) is the Meijer G-function defined in$ Section II-A.

Proof: Corollary 2 is the consequence of (5) and the relationship between the ratio of two independent $\chi^{2}$ random variables and the Fisher $(F)$ distribution with two parameters given as follows.

1) If $X \sim \chi^{2}\left(d_{1}\right)$ and $Y \sim \chi^{2}\left(d_{2}\right)$ are two independent random variables, then $\left(\left(X / d_{1}\right) /\left(Y / d_{2}\right)\right) \sim \mathrm{F}\left(d_{1}, d_{2}\right)$.

2) If $X \sim \mathrm{F}(2 \alpha, 2 \beta)$ has an F-distribution, then $(\alpha / \beta) X \sim$ $\operatorname{Beta}^{\mathrm{II}}(\alpha, \beta)$.

\section{A. Meijer G-Function}

The Meijer G-function is a generalization of the generalized hypergeometric function that is defined using the contour integral representation [25]

$$
\begin{aligned}
G_{p, q}^{m, n} & \left(\begin{array}{c}
a_{1}, \ldots, a_{p} \\
b_{1}, \ldots, b_{q}
\end{array} \mid x\right) \\
= & \frac{1}{2 \pi i} \times \int_{\mathcal{L}} \frac{\prod_{j=1}^{m} \Gamma\left(b_{j}-s\right) \prod_{j=1}^{n} \Gamma\left(1-a_{j}+s\right)}{\prod_{j=1+m}^{q} \Gamma\left(1-b_{j}+s\right) \prod_{j=1+n}^{p} \Gamma\left(a_{j}-s\right)} \\
& \times x^{s} \mathrm{~d} s
\end{aligned}
$$

where $\left\{a_{j}\right\}_{j=1}^{p}$ and $\left\{b_{j}\right\}_{j=1}^{q}$ are, in general, complex-valued. Contour $\mathcal{L}$ is a suitable integration contour that separates the poles of function $\Gamma\left(b_{j}-s\right)$ from the poles of $\Gamma\left(1-a_{j}+s\right)$. The Meijer G-function has been implemented in some commercial software packages, such as MATLAB and MAPLE.

\section{B. Wilks' Lambda Distribution of Determinant Ratio Statistic}

Definition 3: For noninteger values of $n$ and $q$ and integer values of $p$ with $n \geq q \geq p$, the pdf of Wilks's lambda distribution of the second kind, denoted as $\Lambda^{\prime}(n, p, q)$, is given as [17]

$$
f(x)=A^{\prime} \times G_{p, p}^{p, p}\left(\begin{array}{c}
-q,-(q-1), \ldots,-(q-(p-1)) \\
n-q-1, n-q-2, \ldots, n-q-p
\end{array} \mid x\right)
$$

where $A^{\prime}=\prod_{j=0}^{p-1}(1 /(\Gamma(n-q-j) \Gamma(q-j)))$.

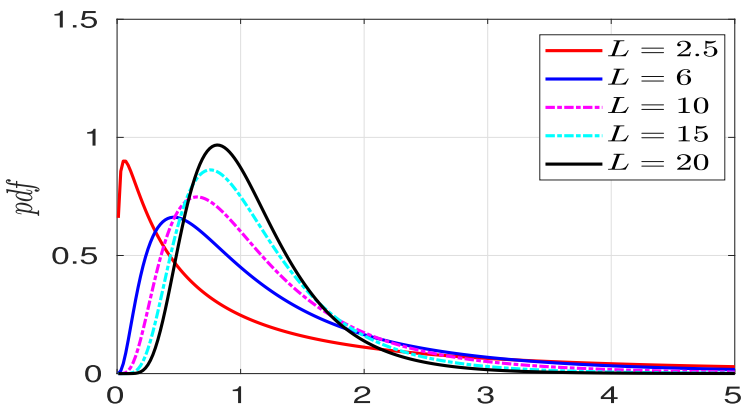

(a)

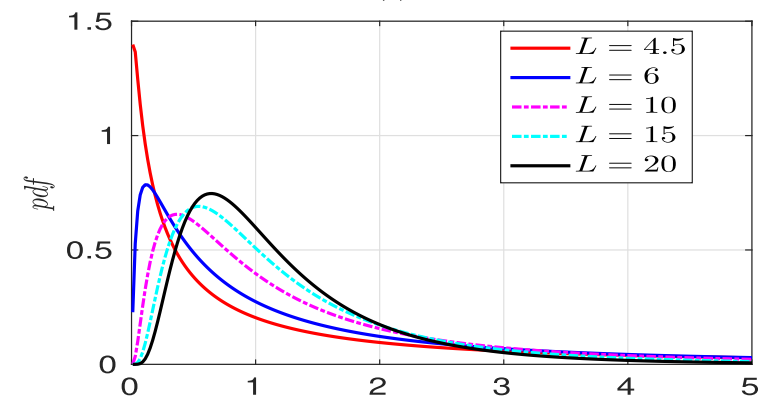

(b)

Fig. 1. PDF of Wilks's lambda distribution of second kind, $\Lambda^{\prime}(2 L, d, L)$ with several values of $L$ and different cases. (a) $d=2$. (b) $d=4$.

Theorem 4: The pdf $g(z)$ of the product $Z=\prod_{i=0}^{d-1} X_{i}$ of $d$ independent $X_{i}$ variables Beta distributed of the second kind, $X_{i} \sim \operatorname{Beta}^{I I}\left(L_{x}-i, L_{y}-i\right)$, is a Meijer G-function multiplied by a normalizing constant $A$, i.e.,

$$
g(z)=A \times G_{d, d}^{d, d}\left(\begin{array}{c}
-L_{y},-\left(L_{y}-1\right), \ldots,-\left(L_{y}-(d-1)\right) \\
L_{x}-1, L_{x}-2, \ldots, L_{x}-d
\end{array} \mid z\right)
$$

where $A=\prod_{i=0}^{d-1}\left(1 /\left(\Gamma\left(L_{x}-i\right) \Gamma\left(L_{y}-i\right)\right)\right)$.

The cumulative distribution function is given by

$$
\begin{aligned}
& G(z)=A z \times \\
& G_{d+1, d+1}^{d, d+1}\left(\begin{array}{c}
-L_{y},-\left(L_{y}-1\right), \ldots,-\left(L_{y}-(d-1)\right), 0 \\
L_{x}-1, L_{x}-2, \ldots, L_{x}-d,-1
\end{array} \mid z\right) .
\end{aligned}
$$

The $k$ th moment $E\left\{Z^{k}\right\}$ is given by

$$
E\left\{Z^{k}\right\}=\prod_{i=0}^{d-1} \frac{\Gamma\left(L_{y}-i-k\right)}{\Gamma\left(L_{y}-i\right)} \frac{\Gamma\left(L_{x}-i+k\right)}{\Gamma\left(L_{x}-i\right)} .
$$

Proof: See Appendix B.

With reference to Definition 3, the distribution of $\left(\left(\left|L_{x} \mathbf{X}\right|\right) /\left(\left|L_{y} \mathbf{Y}\right|\right)\right)$ is given as follows:

$$
\frac{\left|L_{x} \mathbf{X}\right|}{\left|L_{y} \mathbf{Y}\right|} \sim \Lambda^{\prime}\left(L_{x}+L_{y}, d, L_{y}\right) .
$$

It is worthy to note that the determinant ratio depends on the parameters $L_{x}$ and $L_{y}$. In the case $L_{x}=L_{y}=L$, Wilks's lambda distribution of the second kind is given by $\Lambda^{\prime}(2 L, d, L)$. Fig. 1(a) and (b) shows the pdf of $\Lambda^{\prime}(2 L, d, L)$, with multiple values of $L$, and different cases: $d=2$ and $d=4$, respectively.

\section{ACCEPTED MANUSCRIPT}




\section{Polarimetric Change Detector}

We consider $\mathbf{X}$ and $\mathbf{Y}$ two statistically independent Hermitian positive definite random $d \times d$ matrices that follow scaled complex Wishart distributions with different distribution parameters defined as:

$$
\mathbf{X} \sim \operatorname{sW}_{d}^{\mathbb{C}}\left(L_{x}, \boldsymbol{\Sigma}_{x}\right) \text { and } \mathbf{Y} \sim s \mathcal{W}_{d}^{\mathbb{C}}\left(L_{y}, \boldsymbol{\Sigma}_{y}\right) .
$$

Multilook PolSAR images acquired over the same geographical area before event (at time $t_{x}$ ) and after event (at time $t_{y}$ ) are used to detect any change by comparing at each position $(i, j)$ the full polarimetric information before change and after change given, respectively, by $\mathbf{X}(i, j)$ and $\mathbf{Y}(i, j)$. Here, we suppose that at each $(i, j)$, we have two matrices $\mathbf{X}(i, j)$ and $\mathbf{Y}(i, j)$. As a consequence, we resort to compute the determinant ratio of $L_{x} \mathbf{X}$ and $L_{y} \mathbf{Y}$ described in Section IV-A.

\section{A. Determinant Ratio Statistic}

The determinant ratio statistic is defined by

$$
\tau_{\text {DRT }}=\frac{\left|L_{x} \mathbf{X}\right|}{\left|L_{y} \mathbf{Y}\right|} .
$$

DRT is used to measure the similarity between the two polarimetric covariance matrices $\mathbf{X}$ and $\mathbf{Y}$ and perform change detection by choosing between hypotheses [5]

$$
\begin{cases}H_{0}: & \boldsymbol{\Sigma}_{x}=\boldsymbol{\Sigma}_{y} \\ H_{1}: & \boldsymbol{\Sigma}_{x} \neq \boldsymbol{\Sigma}_{y} .\end{cases}
$$

Null hypothesis $\left(H_{0}\right)$ corresponds to no-change, and hypothesis $\left(H_{1}\right)$ corresponds to change. To quantify the difference between $H_{0}$ and $H_{1}$, a threshold selection procedure is applied to the test statistic $\tau_{\mathrm{DRT}}$. It is worth mentioning that the hypothesis tests are developed with distinct ENLs, i.e., $L_{x} \neq L_{y}$. Nevertheless, in the first experiment with simulations, we assume that the ENL is the same for both images.

The exact distribution of the DRT statistic under null hypothesis $\left(H_{0}\right)$ is Wilks's lambda distribution of the second kind given by

$$
\tau_{\mathrm{DRT}} \sim \Lambda^{\prime}\left(L_{x}+L_{y}, d, L_{y}\right) .
$$

For particular case where $L_{x}=L_{y}=L$, the DRT statistic becomes $\tau_{\mathrm{DRT}} \sim \Lambda^{\prime}(2 L, d, L)$.

\section{B. CFAR Thresholding Method}

In our study, change detection is realized by applying a decision threshold to the test statistics [5]. We choose the CFAR algorithm [15] as a thresholding method in order to perform a fair comparison between our proposed approach and the HLT method since we are interested in the test statistic rather than the thresholding method. Let $f_{\tau_{\mathrm{DRT}}}(\tau)$ be the distribution of the ratio $\tau_{\mathrm{DRT}}$ under the hypothesis $H_{0}$. The significance level of the test $\alpha_{c}$, expressed in percent, is given as a function of the desired false alarm probability $P_{\mathrm{fa}}$ and then given by $\alpha_{c}=100 P_{\mathrm{fa}}$. The threshold is determined from the distribution of the determinant ratio statistic.
We adopt the same approach presented in the paper of Akbari et al. [5] where changes from $\mathbf{X}$ to $\mathbf{Y}$ and reversely from $\mathbf{Y}$ to $\mathbf{X}$ were considered in the CFAR change detector. This results in using the following two ratios:

$$
\tau_{\mathrm{DRT}}=\frac{\left|L_{x} \mathbf{X}\right|}{\left|L_{y} \mathbf{Y}\right|} \text {, and } \tau_{\mathrm{DRT}}^{\prime}=\frac{\left|L_{y} \mathbf{Y}\right|}{\left|L_{x} \mathbf{X}\right|} \text {. }
$$

The combined test is given by

$$
\max \left\{\tau_{\mathrm{DRT}}, \tau_{\mathrm{DRT}}^{\prime}\right\} \underset{H_{0}}{\stackrel{H_{1}}{\gtrless}} T .
$$

The combined threshold $T$ is derived from

$$
P_{\mathrm{fa}}=2 \int_{T}^{+\infty} f_{\tau_{\mathrm{DRT}}}\left(\tau \mid H_{0}\right) \mathrm{d} \tau .
$$

When $P_{\mathrm{fa}}$ is specified, the threshold is obtained by solving (22), and then, the CFAR change detector is obtained. The proposed unsupervised change detection based on DRT between two multilook PolSAR data acquired before and after change is summarized in the following steps.

1) Find a global estimation of $\hat{L}_{x}$ and $\hat{L}_{y}$.

2) Generate the DRT statistics image using $\max \left\{\tau_{\mathrm{DRT}}, \tau_{\mathrm{DRT}}^{\prime}\right\}$ computed from two multilook PolSAR data.

3) Compute the CFAR threshold for a specific $P_{\mathrm{fa}}$.

4) Apply the threshold and obtain the binary change detection map.

It is important to mention that the quality of the change detection map depends on the estimation accuracy of $L_{x}$ and $L_{y}$. For an efficient estimation of the ENL, many methods have been put forward in the literature for automatic estimate. To name a few of them, we mention the following. First, the method of Anfinsen et al. [16] was based on the maximum likelihood estimator for the ENL under the assumption that data follow the complex Wishart distribution. Second, the method proposed by Tao et al. [26] was based on the development of trace moments (DTMs). This ENL estimator cancels the textural variation using trace moments. Finally, the method developed by Bouhlel [27] was based on the fractional moments of the determinant of the multilook polarimetric covariance (FMDC) matrix. The FMDC estimator of the ENL had the particularity of being independent of the distribution of the texture model. All these methods consisted in performing a local estimate of the ENL by using a sliding window covering the whole image. Then, the distribution of estimates was drawn and the mode value of the density corresponds to the desired global estimate of the ENL. It is worthy to note that the presence of texture and correlation between samples can affect the estimation of the ENL.

In Section IV-C, we briefly describe the HLT and the LRT statistics in order to compare them with our proposed statistic.

\section{HLT Statistic}

The complex-kind HLT statistic is defined as

$$
\tau_{\mathrm{HLT}}=\operatorname{tr}\left(\mathbf{Y}^{-1} \mathbf{X}\right) .
$$

The exact distribution of the HLT statistic is difficult to derive and an approximation was put forward by Akbari et al. [5]. 
It is the $\mathrm{FS}^{1}$ distribution with three parameters used as an approximation to $\tau_{\mathrm{HLT}}$ and is given as follows:

$$
\tau_{\mathrm{HLT}} \sim \mathrm{FS}(\xi, \zeta, \mu) .
$$

The expression of the FS distribution is given by

$$
f_{\tau_{\mathrm{HLT}}}(\tau)=\frac{\Gamma(\zeta+\xi)}{\Gamma(\xi) \Gamma(\zeta)} \frac{\xi}{\mu(\zeta-1)} \frac{\left(\frac{\xi \tau}{\mu(\zeta-1)}\right)^{\xi-1}}{\left(1+\frac{\xi \tau}{\mu(\zeta-1)}\right)^{\xi+\zeta}}
$$

where $\mu=E\left\{\tau_{\mathrm{HLT}}\right\}>0$ is a scale parameter and $\xi, \zeta>0$ are two shape parameters. The parameters of the FS distribution are computed in terms of distribution parameters of scaled Wishart matrices $\mathbf{X}$ and $\mathbf{Y}$. The solutions for $\mu, \xi$, and $\zeta$ are defined by the following equation system [5]:

$$
m_{v}^{(\mathrm{FS})}(\mu, \xi, \zeta)=m_{v}^{(\mathrm{HLT})}\left(L_{x}, L_{y}, d\right), \quad v=1,2,3
$$

where $m_{v}^{(\mathrm{FS})}$ and $m_{v}^{(\mathrm{HLT})}$ are the $v$ th order moments of the FS distribution and the HLT statistic, respectively. For more details about the expressions of the moments, reader can refer to [5] and [28]. Technically, to estimate the shape parameters $\xi$ and $\zeta$, a minimum distance optimization is used to solve the following:

$$
(\hat{\xi}, \hat{\zeta})=\arg \min _{(\xi, \zeta)} \sum_{v=2}^{3}\left(m_{v}^{(\mathrm{FS})}-m_{v}^{(\mathrm{HLT})}\right)^{2} .
$$

Finally, a good fitting of the FS distribution depends on the estimation accuracy of $(\mu, \xi, \zeta)$, which in turn depends on the estimates of $L_{x}$ and $L_{y}$ [5]. It is worth noticing that the FS distribution of the HLT detector for a low number of looks does not provide a good approximation and fitting as it will be seen in the experiments.

The combined test is derived as follows:

$$
\max \left\{\tau_{\mathrm{HLT}}, \tau_{\mathrm{HLT}}^{\prime}\right\} \underset{H_{0}}{\stackrel{H_{1}}{\gtrless}} T \text {. }
$$

The threshold is determined from the resolution of the following equation:

$$
P_{\mathrm{fa}}=2 \int_{T}^{+\infty} f_{\tau_{\mathrm{HLT}}}\left(\tau \mid H_{0}\right) \mathrm{d} \tau
$$

\section{LRT Statistic}

The Wishart LRT statistic was derived as [10]

$$
Q=\frac{\left(L_{x}+L_{y}\right)^{d\left(L_{x}+L_{y}\right)}}{L_{x}^{d L_{x}} L_{y}^{d L_{y}}} \frac{\left|L_{x} \mathbf{X}\right|^{L_{x}}\left|L_{y} \mathbf{Y}\right|^{L_{y}}}{\left|L_{x} \mathbf{X}+L_{y} \mathbf{Y}\right|^{L_{x}+L_{y}}} .
$$

The test statistic for change detection based on the LRT is given by

$$
\tau_{\mathrm{LRT}}=-2 \rho \ln Q
$$

where

$$
\rho=1-\frac{2 d^{2}-1}{6 d}\left(\frac{1}{L_{x}}+\frac{1}{L_{y}}-\frac{1}{L_{x}+L_{y}}\right) .
$$

\footnotetext{
${ }^{1}$ This notation is used for FS distribution with three parameters. However, with two parameters, we use the notation (F).
}

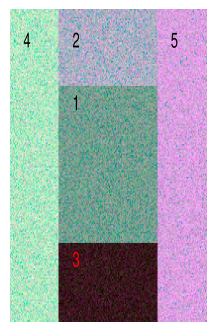

(a)

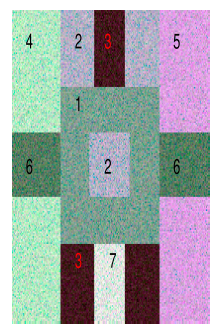

(b)

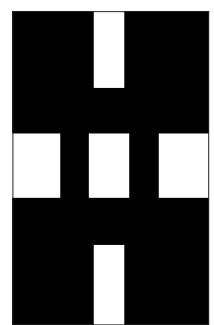

(c)
Fig. 2. Pauli decomposition of simulated five-look quad-pol PolSAR data. (a) Before change. (b) After change. (c) Binary truth change map.

The distribution of $\tau_{\mathrm{LRT}}$ under the null hypothesis $\left(H_{0}\right)$ is approximated by using the associated asymptotic distribution of the test statistic [10]

$$
\tau_{\mathrm{LRT}} \sim \chi^{2}\left(d^{2}\right)+w_{2}\left[\chi^{2}\left(d^{2}+4\right)-\chi^{2}\left(d^{2}\right)\right]
$$

where $\chi^{2}\left(d^{2}\right)$ denotes a central $\chi^{2}$ distribution with $d^{2}$ degrees of freedom and

$$
\begin{aligned}
w_{2}=-\frac{d^{2}}{4}\left(1-\frac{1}{\rho}\right)^{2} & +\frac{d^{2}\left(d^{2}-1\right)}{24} \\
& \times\left(\frac{1}{L_{x}^{2}}+\frac{1}{L_{y}^{2}}-\frac{1}{\left(L_{x}+L_{y}\right)^{2}}\right) \frac{1}{\rho^{2}} .
\end{aligned}
$$

The test with a desired $P_{\mathrm{fa}}$ is given by

$$
\tau_{\text {LRT }} \underset{H_{0}}{\stackrel{H_{1}}{\gtrless}} T
$$

where the threshold $T$ is determined through the equation

$$
P_{\mathrm{fa}}=\int_{T}^{+\infty} f_{\tau_{\mathrm{LRT}}}\left(\tau \mid H_{0}\right) \mathrm{d} \tau \text {. }
$$

It is worth mentioning that the LRT is a one-sided test; however, the DRT and HLT are two-sided test.

\section{EXPERIMENTAL RESUlTS}

The performance of the proposed DRT statistic is evaluated on both simulated and real PolSAR images. The HLT and the LRT tests are implemented for comparison with our test.

\section{A. Simulated Data}

We simulate two quad-pol data containing two $L$-look PolSAR images of $250 \times 250$ pixels and having four polarimetric channels $(d=4)$. The generated data follow a scaled Wishart distribution with a covariance matrix of the speckle $\boldsymbol{\Sigma}_{j}$ defined in Table I where $j=\{1, \ldots, 7\}$. Then, the polarimetric data contain seven different classes (areas). Area 7 corresponds to the polarimetric properties of a heterogeneous urban area. Area 3 is simulated with the polarimetric properties of a homogeneous water region. The rest of the areas correspond to the properties of agricultural crops and vegetation regions. Different values of the number of looks $L$ are used in this study, $L \in\{5,6,7,8\}$. Fig. 2 shows the Pauli decomposition of the two simulated five-look quad-pol PolSAR data corresponding to images before and after change and the binary 


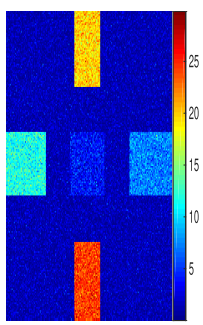

(a1)

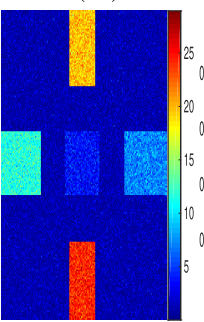

(a2)

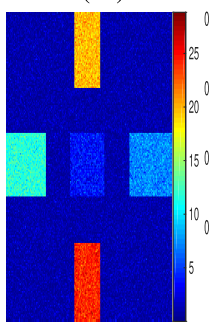

(a3)

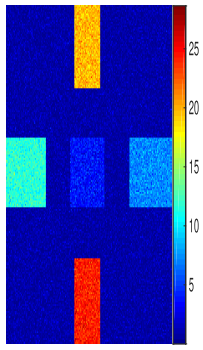

(a4)

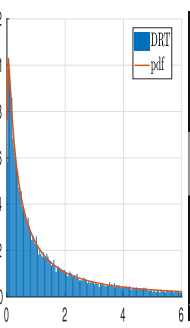

(b1)

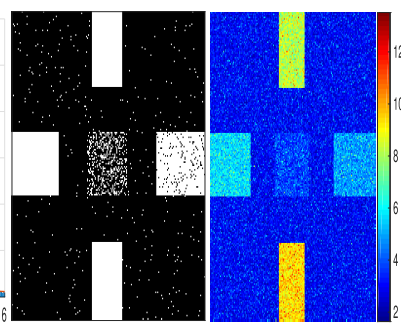

(c1)

$\left(\mathrm{al}{ }^{\prime}\right)$

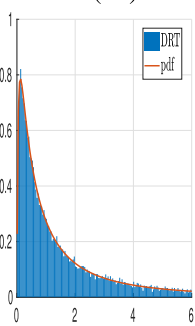

(b2)
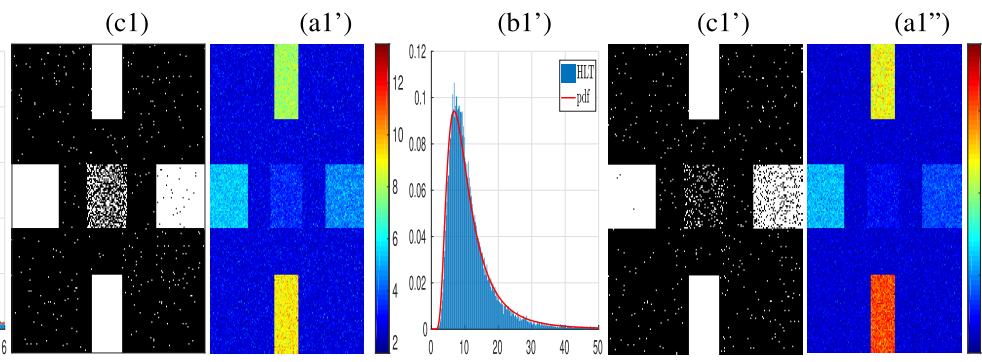

(a2')

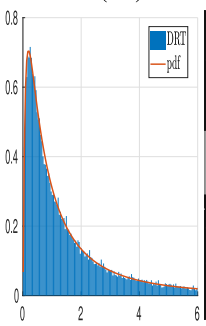

(c2)

(b3)
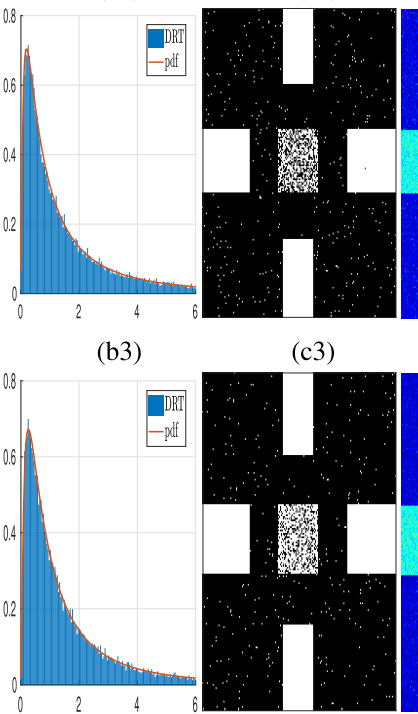

(b4)

(c3)

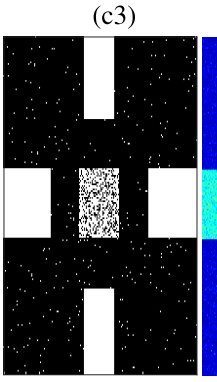

(c4)

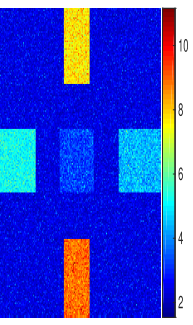

(a3')

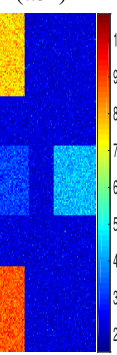

(a4')

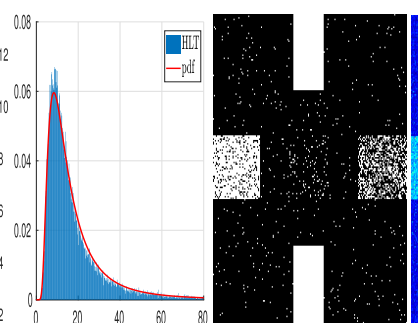

(c1')

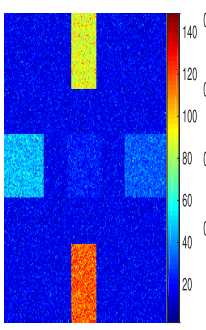

(a1")

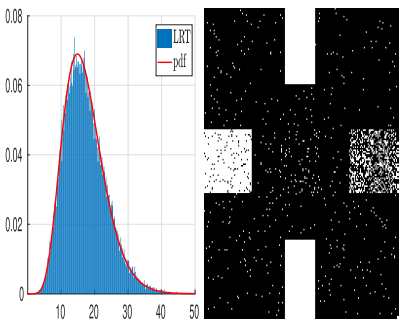

(b1") (c1")

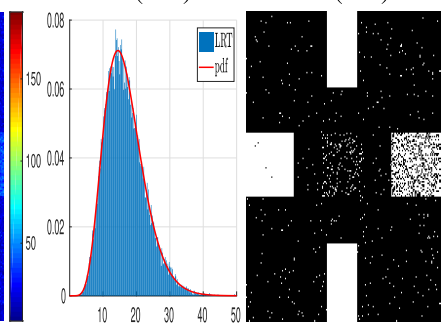

(b2')

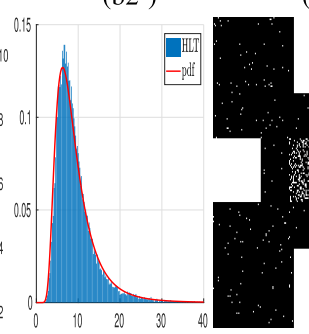

(c2')

(a2")

(b2")

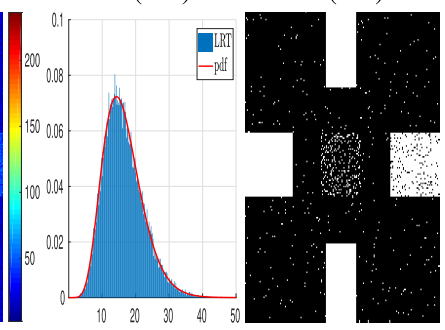

(b3')

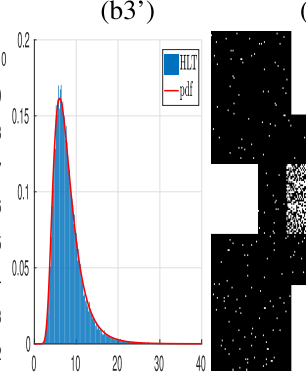

(b4')

(c4')

(b3")

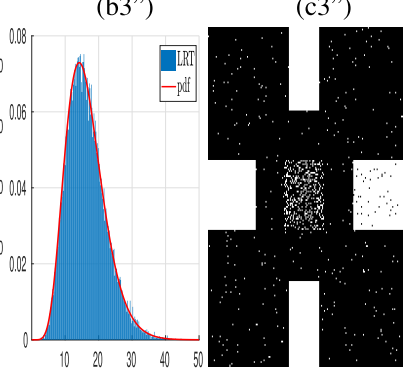

(b4")

(c4”)

Fig. 3. Comparison between DRT, HLT, and LRT statistics for simulated data with different levels of multilooking. The number of looks is $L \in\{5,6,7,8\}$, where the first raw $L=5$ and the last raw $L=8$. (ai) Logarithm of $\max \left\{\tau_{\mathrm{DRT}}, \tau_{\mathrm{DRT}}^{\prime}\right\}$. (bi) Plots of $\tau_{\mathrm{DRT}}$ normalized histograms and estimated Wilk's lambda of second kind pdfs under $\left(H_{0}\right)$ hypothesis. (ai') Logarithm of $\max \left\{\tau_{\mathrm{HLT}}, \tau_{\mathrm{HLT}}^{\prime}\right\}$. (bi') Plots of $\tau_{\mathrm{HLT}}$ normalized histograms and estimated FS pdfs under $\left(H_{0}\right)$ hypothesis. (ai") $\tau_{\text {LRT }}$. (bi") Plots of $\tau_{\text {LRT }}$ normalized histograms and estimated distribution given by (33) under $\left(H_{0}\right)$ hypothesis. Change detection map obtained by rejection of hypothesis test at $1 \%$ significance level for (ci) DRT, (ci') HLT, and (ci') LRT detector.

TABLE I

Covariance Matrix For EACH Region of Simulated Data

\begin{tabular}{|c|c|}
\hline Area & $\left\{\Sigma_{11}, \Sigma_{22}, \Sigma_{33}, \Sigma_{44}, \Sigma_{12}, \Sigma_{13}, \Sigma_{14}, \Sigma_{23}, \Sigma_{24}, \Sigma_{34}\right\} \times 10^{-3}$ \\
\hline \hline 1 & $2.6,0.6,0.6,2.9,0,0,0.9-1.2 \mathrm{i}, 0,0,0$ \\
\hline 2 & $11.9,1,1,7.7,0,0,-2.1-3.6 \mathrm{i}, 1,0,0,0$ \\
\hline 3 & $0.28,0.007,0.007,0.073,0,0,0.13-0.004 \mathrm{i}, 0,0,0$ \\
\hline 4 & $6.7,6,6,11.2,0,0,2.2+0.8 \mathrm{i}, 0,0,0$ \\
\hline 5 & $27.3,0.6,0.6,12,0,0,14.2-6.4 \mathrm{i}, 0,0,0$ \\
\hline 6 & $1,0.2,0.2,0.8,0,0,0.5-\mathrm{i}, 0,0,0$ \\
\hline 7 & $8.9,5.5,5.5,26.1,0,0,-1.1+0.2 \mathrm{i}, 0,0,0$ \\
\hline
\end{tabular}

truth change map where the change is marked by the white and the no-change by the black.

By computing the DRT, HLT, and LRT statistics, Fig. 3 shows the results of change detection relative to these methods and for different cases of $L$. As it can be seen, each row corresponds to a particular value of $L$ starting from 5 for the first row to 8 for the last row. Fig. 3(ai), (ai'), and (ai") where $i \in\{1,2,3,4\}$ show, respectively, the logarithm of DRT, the logarithm of HLT, and the LRT statistics for different values of $L$. In addition, Fig. 3(bi) shows the comparison between the normalized histograms of $\tau_{\mathrm{DRT}}$ and the estimated Wilks's lambda of the second kind pdfs computed over the nonchange area. It is clear that the estimated pdf curves fit well with the normalized histograms. The same comparison is made in Fig. 3(bi') between $\tau_{\text {HLT }}$ and the estimated FS pdfs used as approximations. It is shown that for a low number of looks smaller than 7, the FS distribution of the HLT detector is not providing a good fitting. However, the DRT detector works better for the small number of looks. 
TABLE II

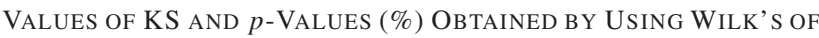
SECOND Kind Distribution For Simulated Data With DIFFERENT LEVELS OF MULTILOOKING

\begin{tabular}{|c|c|c|c|c|}
\hline & $L=5$ & $L=6$ & $L=7$ & $L=8$ \\
\hline \hline KS & 0.0039 & 0.0033 & 0.0028 & 0.0022 \\
\hline$p$-value & 0.61 & 0.75 & 0.89 & 0.98 \\
\hline
\end{tabular}

Another comparison is made in Fig. 3(bi") between $\tau_{\text {LRT }}$ and the estimated pdf given by (33). Furthermore, Fig. 3(ci), (ci'), and (ci") shows the corresponding binary change maps obtained by the thresholding CFAR algorithm. Indeed, binary change detection map is obtained by the rejection of the hypothesis test at a $1 \%$ significance level for the DRT, HLT, and LRT detectors. It is noted that changes can be seen in these statistics for each case of $L$-values. Moreover, the proposed method detects more efficiently the changed area even for highly speckled cases with a low number of looks. It is also worth noticing that at the central part of the binary images (region 2), where heterogeneous change detection is present, the DRT is better than the others, but all three detectors seem not to work properly. This leads us to extend this work under non-Wishart case [29], [30] or relaxed Wishart distribution where the ENL is a local parameter [31].

The fit ability between the estimated Wilks's of the second kind pdfs and the normalized histograms of $\tau_{\mathrm{DRT}}$ is evaluated qualitatively by using the Kolmogorov-Smirnov (KS) hypothesis test. The smaller value of KS indicates better the hypothesized model fits with the empirical distribution. A small value of $p$-value of the test indicates strong incompatibilities of the data with the employed distribution hypothesis. Table II lists the values of KS and the $p$-values of the test (in percentage) obtained for the four cases of $L$ under null hypothesis $\left(H_{0}\right)$. It is evident from the $p$-value that the fitted Wilk's of the second kind distribution can perfectly model the no-change area.

A quantitative evaluation of the change detection performance is also provided at four different significance levels or specified FARs. Table III shows the measured FAR [false positive rate (FPR)] and the detection rate [true positive rate (TPR)] for DRT, HLT, and LRT statistics for various levels of multilooking and for different specified FARs. As shown in Table III, the DRT statistic realizes higher detection rates and lower overall error rates than the HLT and LRT statistics at specified FARs, especially when the speckle is strong and the number of looks $L$ is low. As $L$ increases to reach 8 and at specified FARs, the detection rates for these statistical tests increase and the overall error rates decrease. Likewise, when $L$ passes to 8 , the detection rate of both the DRT and HLT becomes close. It is also worth noticing that the measured FAR is close to the specified FAR regardless of the detector used.

Another quantitative evaluation of the performance of the method is provided by the receiver operating characteristic (ROC) curves that are plotted for these statistics using the ground truth. The ROC curve is the evolution of the TPR as a function of the FPR [32]. Fig. 4 shows the ROC
TABLE III

Change Detection Performance for Simulated Data. The Best VALUES ARE MARKED B Y RED

\begin{tabular}{|c|c|c|c|c|c|}
\hline Looks & $\begin{array}{c}\text { Specified } \\
\text { FAR }(\%)\end{array}$ & Method & $\begin{array}{l}\text { Measured } \\
\text { FAR }(\%)\end{array}$ & $\begin{array}{l}\text { Detection } \\
\text { rate }(\%)\end{array}$ & $\begin{array}{c}\text { Overall error } \\
\text { rate }(\%)\end{array}$ \\
\hline \multirow{12}{*}{5} & \multirow{3}{*}{0.5} & $\overline{\text { DRT }}$ & 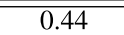 & \begin{tabular}{|c|}
82.20 \\
\end{tabular} & 4.30 \\
\hline & & HLT & 0.76 & 58.27 & 9.86 \\
\hline & & LRT & 0.69 & 62.26 & 8.92 \\
\hline & \multirow{3}{*}{1} & DRT & 0.92 & 85.08 & 4.03 \\
\hline & & HLT & 1.40 & 65.66 & 8.71 \\
\hline & & LRT & 1.23 & 66.51 & 8.39 \\
\hline & \multirow{3}{*}{5} & DRT & 5.05 & 90.99 & 5.93 \\
\hline & & HLT & 5.15 & 80.11 & 8.43 \\
\hline & & LRT & 5.58 & 77.58 & 9.31 \\
\hline & \multirow{3}{*}{10} & DRT & 10.00 & 93.78 & 9.16 \\
\hline & & HLT & 9.56 & 85.74 & 10.61 \\
\hline & & LRT & 10.89 & 82.97 & 12.25 \\
\hline \multirow{12}{*}{6} & \multirow{3}{*}{0.5} & $\overline{\text { DRT }}$ & 0.54 & $\begin{array}{l}87.40 \\
\end{array}$ & 3.21 \\
\hline & & HLT & 0.68 & 75.36 & 6.00 \\
\hline & & LRT & 0.52 & 72.47 & 6.51 \\
\hline & \multirow{3}{*}{1} & DRT & 1.01 & 89.17 & 3.19 \\
\hline & & HLT & 1.16 & 79.28 & 5.50 \\
\hline & & LRT & 1.07 & 75.97 & 6.17 \\
\hline & \multirow{3}{*}{5} & DRT & 4.87 & 94.13 & 5.09 \\
\hline & & HLT & 4.74 & 88.09 & 6.34 \\
\hline & & LRT & 5.35 & 85.04 & 7.48 \\
\hline & \multirow{3}{*}{10} & DRT & 9.72 & 96.23 & 8.40 \\
\hline & & HLT & 9.04 & 91.42 & 8.93 \\
\hline & & LRT & 10.33 & 88.64 & 10.56 \\
\hline \multirow{12}{*}{7} & \multirow{3}{*}{0.5} & $\overline{\text { DRT }}$ & $\overline{0.54}$ & 90.25 & 2.59 \\
\hline & & HLT & 0.57 & 82.82 & 4.26 \\
\hline & & LRT & 0.53 & 79.65 & 4.93 \\
\hline & \multirow{3}{*}{1} & DRT & 1.11 & 92.14 & 2.61 \\
\hline & & HLT & 1.04 & 85.15 & 4.11 \\
\hline & & LRT & 1.05 & 82.15 & 4.78 \\
\hline & \multirow{3}{*}{5} & DRT & 5.16 & 96.44 & 4.80 \\
\hline & & HLT & 4.70 & 92.05 & 5.42 \\
\hline & & LRT & 5.29 & 88.24 & 6.72 \\
\hline & \multirow{3}{*}{10} & DRT & 10.23 & 97.82 & 8.44 \\
\hline & & HLT & 9.09 & 95.06 & 8.17 \\
\hline & & LRT & 10.37 & 91.81 & 9.89 \\
\hline \multirow{12}{*}{8} & \multirow{3}{*}{0.5} & $\overline{\text { DRT }}$ & 0.46 & $\overline{92.86}$ & 1.94 \\
\hline & & HLT & 0.58 & 87.97 & 3.12 \\
\hline & & LRT & 0.44 & 83.23 & 4.07 \\
\hline & \multirow{3}{*}{1} & DRT & 1.00 & 94.60 & 1.98 \\
\hline & & HLT & 1.13 & 90.33 & 3.02 \\
\hline & & LRT & 0.95 & 85.25 & 4.02 \\
\hline & \multirow{3}{*}{5} & DRT & 5.22 & 97.88 & 4.53 \\
\hline & & HLT & 5.07 & 95.79 & 4.88 \\
\hline & & LRT & 5.02 & 90.97 & 5.91 \\
\hline & \multirow{3}{*}{10} & DRT & 10.23 & 98.85 & 8.21 \\
\hline & & HLT & 9.77 & 97.65 & 8.12 \\
\hline & & LRT & 10.18 & 93.89 & 9.27 \\
\hline
\end{tabular}

curves for DRT, HLT, and LRT statistics and for different values of $L$. It is shown that the DRT statistics give the best performance for this example followed by the HLT statistics in all cases and then by the LRT statistics. The area under ROC curve (AUC) is also considered in this study. The larger the AUC, the better the performance. Accordingly, the AUC values are shown in Table IV for these statistics and multiple values of $L$, and we can easily conclude that the proposed DRT statistic outperforms both the HLT and the LRT statistics for low values of $L$. When the ENL increases, the AUC of the HLT reaches the AUC of the DRT statistic. Consequently, the two statistics give us similar results. The performance of the proposed change detection algorithm is further evaluated with different real multilook PolSAR data sets in Section V-B. 


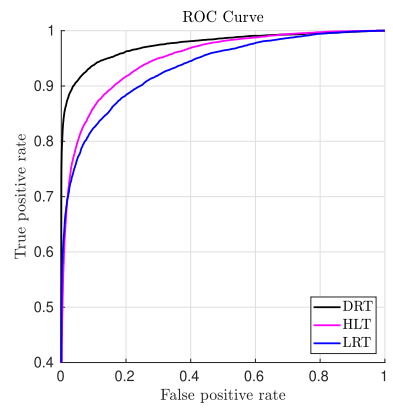

(a)

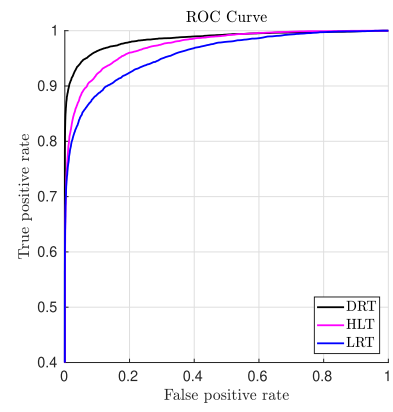

(b)

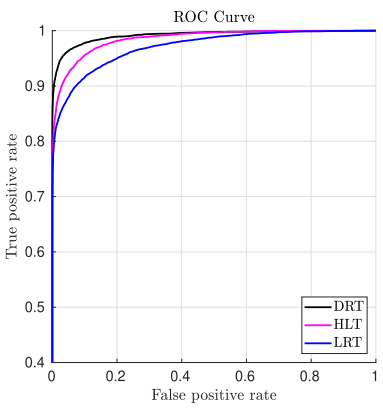

(c)

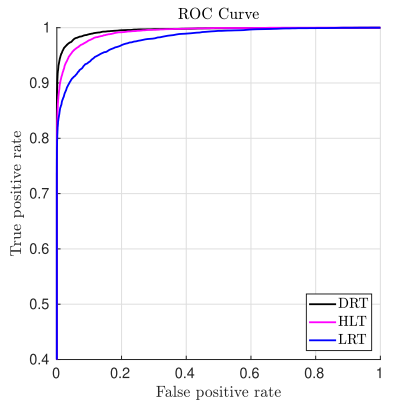

(c)

Fig. 4. ROC curves comparison of three statistical detectors DRT, HLT, and LRT for different levels of multilooking $L \in\{5,6,7,8\}$. The probability of detection (TPR) is plotted as a function of the probability of a false alarm (FPR) for simulation data. The DRT statistic performs better than HLT and LRT statistics. (a) $L=5$. (b) $L=6$. (c) $L=7$. (c) $L=8$.

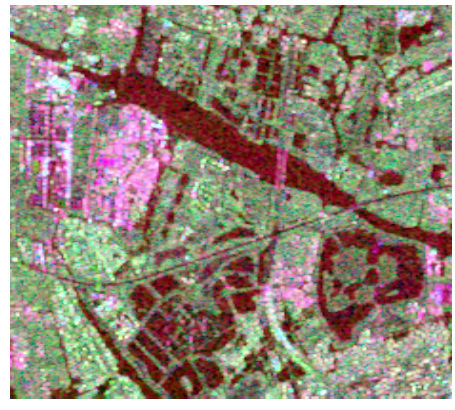

(a)

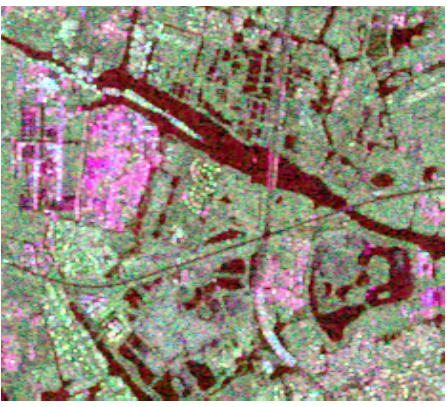

(b)

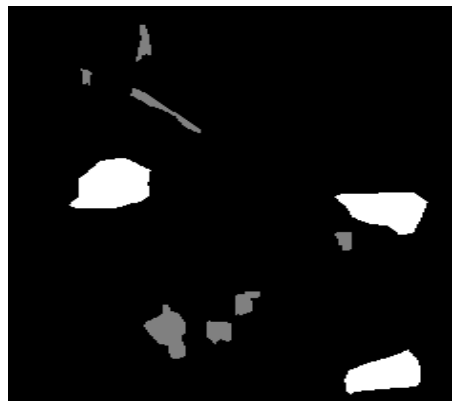

(c)

Fig. 5. Pauli RGB ( $\mathrm{R}=\mathrm{HH}-\mathrm{VV}, \mathrm{G}=\mathrm{HV}$, and $\mathrm{B}=\mathrm{HH}+\mathrm{VV}$ ) of quadpol RADARSAT-2 images captured over Suzhou city, East China on (a) April 9, 2009 and (b) June 15, 2010, multilooked with 24-looks. (c) Truth change map. The white means no-change, the gray means change, and the black means unlabeled pixels.

TABLE IV

AUC FOR Simulated Data COMPUTED With VARious Methods (DRT, HLT, AND LRT) AND VARIOUS NUMBER OF LOOKS. THE BEST VAlues ARe MARKed by Red

\begin{tabular}{|c|c|c|}
\hline Looks & Method & AUC(\%) \\
\hline \multirow{2}{*}{5} & DRT & 97.30 \\
& HLT & 94.95 \\
& LRT & 93.46 \\
\hline \hline \multirow{2}{*}{6} & DRT & 98.52 \\
& HLT & 97.31 \\
& LRT & 95.74 \\
\hline \hline \multirow{2}{*}{7} & DRT & 99.16 \\
& HLT & 98.51 \\
& LRT & 97.11 \\
\hline \hline \multirow{2}{*}{8} & DRT & 99.54 \\
& HLT & 99.20 \\
& LRT & 98.00 \\
\hline
\end{tabular}

\section{B. Real PolSAR Data}

1) Data set 1: For real data, we use a set of two RADARSAT-2 fully PolSAR images over an urban area in Suzhou city, East China, acquired on April 9, 2009, and June 15,2010 . The two images corresponding to before and after changes are multilooked with 24-looks (six looks in range and four looks in azimuth directions). Fig. 5(a) and (b) shows the Pauli decomposition images of these two PolSAR images. The ground-truth map is represented in Fig. 5(c) and contains nochange test pixels with white and change test pixels with gray.
In contrast, the black designates urban areas that do not satisfy the Wishart assumption. The ENL for both images before and after changes is estimated to be 7.2 and 6.9 , respectively. Fig. 6(a), (a'), and (a"), respectively, shows the logarithm of the DRT, the logarithm of the HLT, and the LRT statistic. The plots of the normalized histograms and the estimated distributions for DRT, HLT, and LRT detectors are given, respectively, in Fig. 6(b), (b'), and (b'). Fig. 6(c), (c'), and (c") shows their corresponding binary change map obtained by the thresholding CFAR algorithm. The binary change detection map is obtained by the rejection of the hypothesis test at a $1 \%$ significance level for the DRT, HLT, and LRT detectors.

Regarding the quantitative assessment, the ROC curves for the DRT, HLT, and LRT statistics used in the experiments are given in Fig. 7. In this example, the obtained results show that the ROC curves of the HLT and LRT are above the ROC curve of the DRT. The measured AUC for these methods based on the DTR, HLT, and LRT statistics is $99.68 \%$, $99.45 \%$, and $99.13 \%$, respectively. Different significance levels of the test are chosen where $\alpha_{c} \in\{0.5 \%, 1 \%, 5 \%, 10 \%\}$ for evaluation. The detection rate, the measured FAR, and the overall error rate for the three methods are shown in Table V. At each specified FAR level, the best values are highlighted in red. We can clearly see that the DRT always gives the best detection rate for any specified FAR level. Also, for the overall error rate, the DRT gives good results in comparison with the other statistics for the case of a low value of significance levels

\section{ACCEPTED MANUSCRIPT}




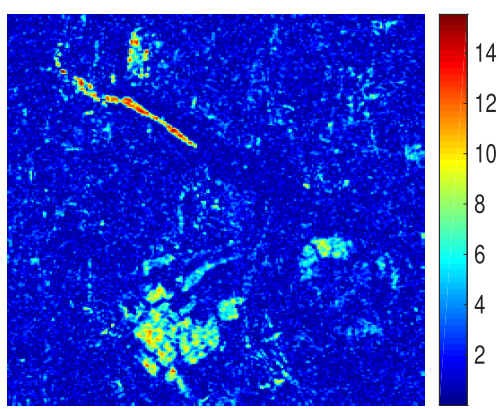

(a) $\ln \left[\max \left\{\tau_{\mathrm{DRT}}, \tau_{\mathrm{DRT}}^{\prime}\right\}\right]$

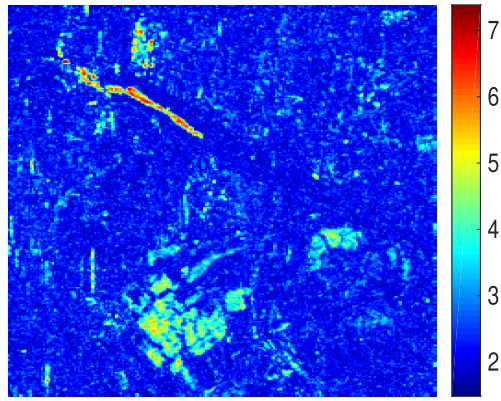

(a') $\ln \left[\max \left\{\tau_{\mathrm{HLT}}, \tau_{\mathrm{HLT}}^{\prime}\right\}\right]$

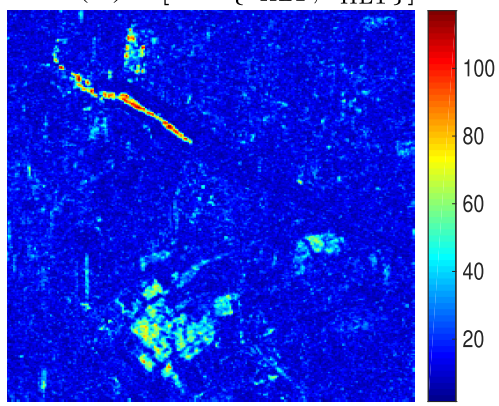

(a”) $\tau_{\text {LRT }}$

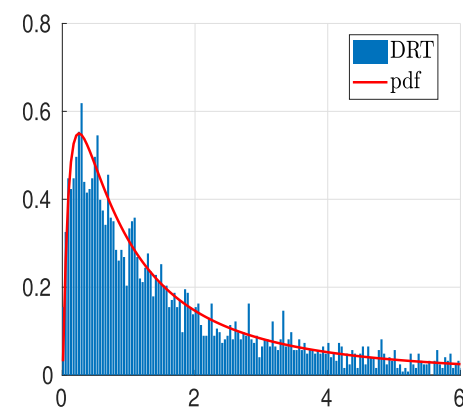

(b)

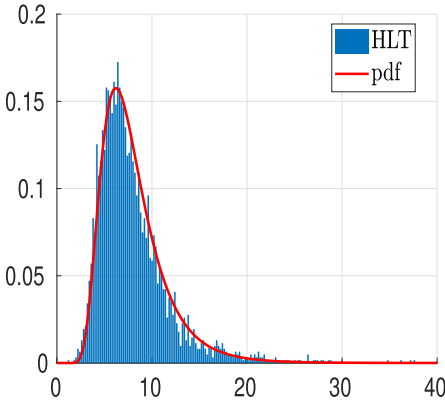

(b')

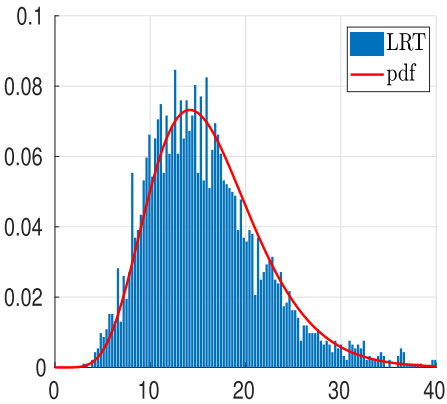

(b")

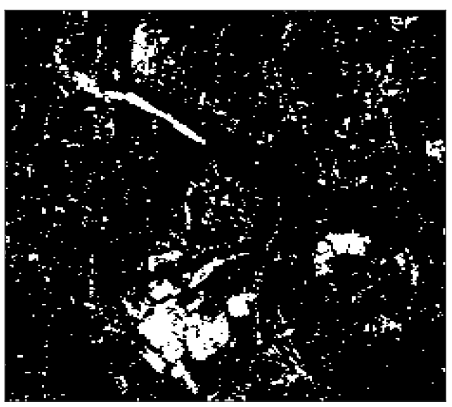

(c) DRT change detection map

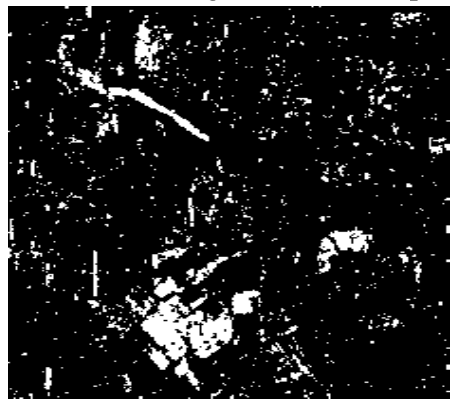

(c') HLT change detection map

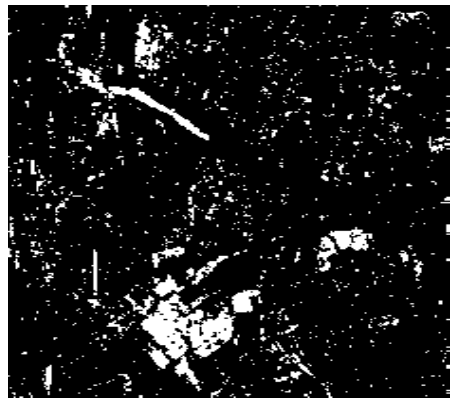

(c") LRT change detection map

Fig. 6. Comparison between DRT, HLT, and LRT statistics for real experimental data set over Suzhou city. (a) Logarithm of max $\left\{\tau_{\mathrm{DRT}}, \tau_{\mathrm{DRT}}^{\prime}\right\}$. (a') Logarithm of $\max \left\{\tau_{\mathrm{HLT}}, \tau_{\mathrm{HLT}}^{\prime}\right\}$. (a") $\tau_{\mathrm{LRT}}$. Plots of normalized histograms and estimated distribution for (b) DRT, (b') HLT, and (b") LRT detectors. Change detection map at $1 \%$ significance level obtained from (c) DRT, (c') HLT, and (c") LRT statistic.

TABLE V

Change Detection Performance for Real Pols ar Data Set 1

\begin{tabular}{|c|c|c|c|c|}
\hline $\begin{array}{c}\text { Specified } \\
\text { FAR(\%) }\end{array}$ & Method & $\begin{array}{c}\text { Measured } \\
\text { FAR }(\%)\end{array}$ & $\begin{array}{c}\text { Detection } \\
\text { rate }(\%)\end{array}$ & $\begin{array}{c}\text { Overall error } \\
\text { rate }(\%)\end{array}$ \\
\hline \hline \multirow{2}{*}{0.5} & DRT & 0.91 & 95.53 & 1.98 \\
& HLT & 0.61 & 90.90 & 3.16 \\
& LRT & 1.11 & 92.50 & 3.03 \\
\hline \multirow{2}{*}{1} & DRT & 1.40 & 96.36 & 2.07 \\
& HLT & 1.10 & 93.56 & 2.71 \\
& LRT & 1.73 & 94.17 & 2.96 \\
\hline \multirow{2}{*}{5} & DRT & 5.24 & 98.63 & 4.07 \\
& HLT & 4.03 & 97.42 & 3.59 \\
& LRT & 5.70 & 97.50 & 4.74 \\
\hline \multirow{2}{*}{10} & DRT & 9.60 & 99.24 & 6.94 \\
& HLT & 7.03 & 98.56 & 5.35 \\
& LRT & 9.77 & 98.41 & 7.31 \\
\hline
\end{tabular}

of the test $\alpha_{c} \in\{0.5 \%, 1 \%\}$. For large values of $\alpha_{c}$, the HLT wins. The measured FAR are close to the specified FAR for the DRT and LRT statistics when $\alpha_{c} \in\{5 \%, 10 \%\}$. However, for low values of $\alpha_{c}$, the HLT gives similar values for measured and specified FAR.

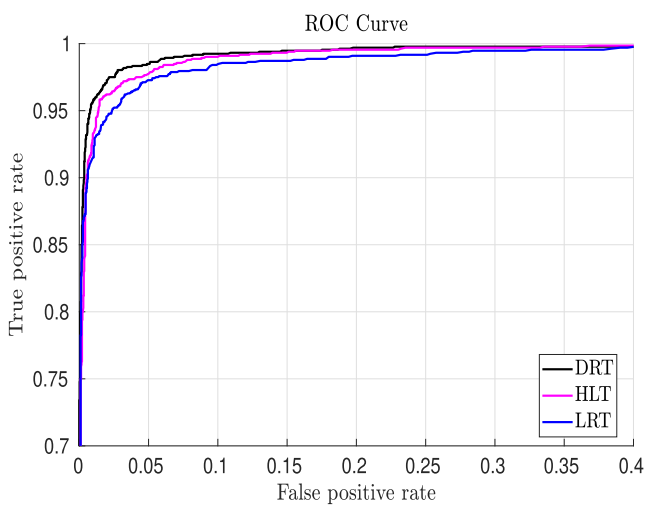

Fig. 7. ROC curves comparison between DRT, HLT, and LRT statistics for real PolSAR data set 1 . The DRT detector performs better than the HLT and LRT detectors.

2) Data set 2: Two coregistered pair of L-band polarimetric images $(d=3)$ corresponding to scenes 1 and 2 were acquired by the Jet Propulsion Laboratory/National Aeronautics and Space Administration UAVSAR $(1.26 \mathrm{GHz})$ over the city of

\section{ACCEPTED MANUSCRIPT}




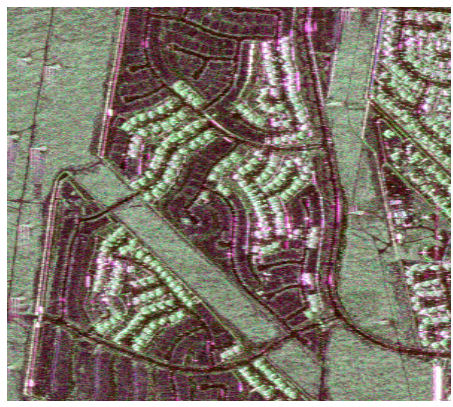

(a)

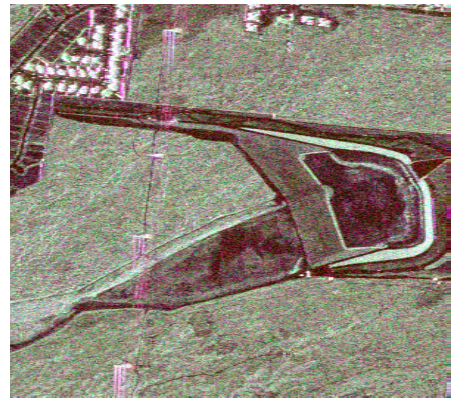

(a')

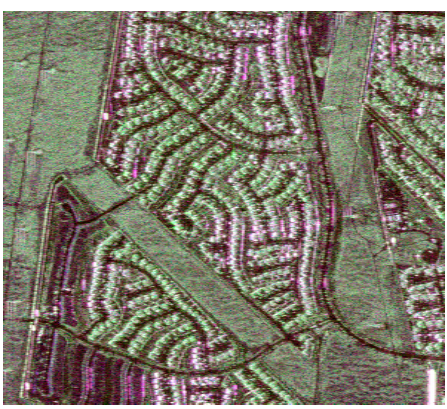

(b)

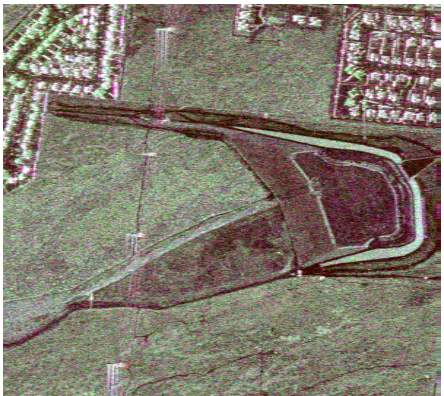

(b')

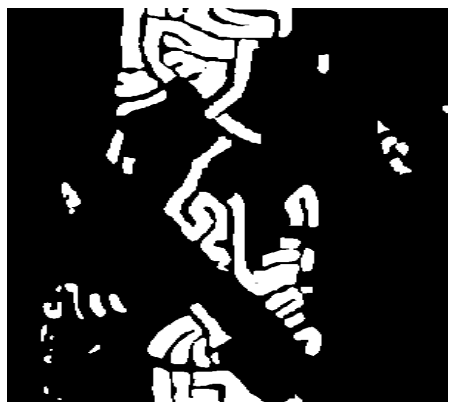

(c)

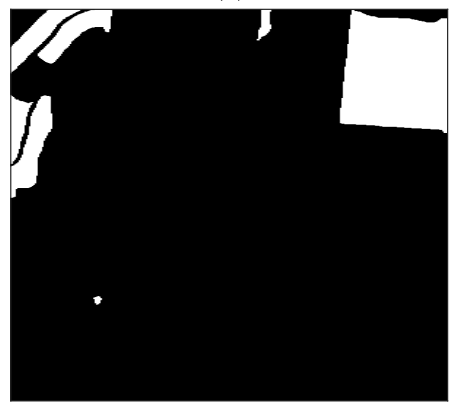

(c')

Fig. 8. UAVSAR images (in Pauli decomposition) over the city of Los Angeles, California. (a) and (a') April 23, 2009. (b) and (b') May 11, 2015. (c) and (c') ground truths, where white is change and black is no-change. Top: Scene 1. Bottom: Scene 2.

Los Angeles, California, on April 23, 2009, and May 11, 2015. The images are $2 \times 3$ multilooked. Fig. 8 shows the Pauli decomposition of these two scenes with two images each are obtained by the JPL's UAVSAR sensor at two different times. Fig. 8(c) and (c') shows the ground truths used to compute the ROC curves. We recall that the white corresponds to change and the black to no-change. As we can see, the interesting area of this data set is an urban area where the changes occurred due to the effects of urbanization.

Fig. 9 compares the change detection results relative to DRT, HLT, and LRT statistics. As it can be noted, the logarithms of $\max \left\{\tau_{\mathrm{DRT}} \tau_{\mathrm{DRT}}^{\prime}\right\}, \max \left\{\tau_{\mathrm{HLT}}, \tau_{\mathrm{HLT}}^{\prime}\right\}$, and $\tau_{\mathrm{LRT}}$ are first computed and shown, respectively, in Fig 9(ai), (ai'), and (ai'), where $i \in\{1,2\}$ representing scenes 1 and 2 . The areas with change are marked in green (small change) and red (strong change). The areas without change are marked in blue. Fig. 9(b1) and (b2) show a good fitting between the normalized histograms of $\tau_{\text {DRT }}$ and the estimated Wilks's lambda of the second kind pdf computed over the nonchange area, respectively. As shown in Section II, Wilks's lambda of the second kind distribution depends on the ENL and dimension $(d)$ of the covariance matrix. The comparison between the normalized histograms of $\tau_{\text {HLT }}$ and the estimated FS pdf computed over the nonchange area is shown in Fig. 9(b1') and (b'2). We can see here a good fitting of the FS distribution with the normalized histograms. The same comparison is realized in Fig. 9(b"1) and (b"2) with the LRT statistic and the approximated pdf given by (33) and computed over the nonchange area. As we can see, the estimated pdf curves based on $\chi^{2}$ distributions do not fit well the normalized histograms. As consequence, the CFAR algorithm fails to provide the best threshold for the LRT statistic. Fig. 9(ci), (ci'), and (ci") shows the binary change
TABLE VI

Change Detection Performance For ReAl PolSAR DATA SET 2: SCENES 1 AND 2

\begin{tabular}{|c|c|c|c|c|}
\hline $\begin{array}{l}\text { Specified } \\
\text { FAR }(\%)\end{array}$ & Methods & $\begin{array}{l}\text { Measured } \\
\text { FAR }(\%)\end{array}$ & $\begin{array}{c}\text { Detection } \\
\text { rate }(\%)\end{array}$ & $\begin{array}{c}\text { Overall error } \\
\text { rate }(\%)\end{array}$ \\
\hline \multicolumn{5}{|c|}{ Scene 1} \\
\hline \multirow{3}{*}{0.5} & DRT & 1.71 & 40.77 & 10.65 \\
\hline & HLT & 0.40 & 18.57 & 12.99 \\
\hline & LRT & 0.41 & 20.26 & 12.74 \\
\hline \multirow{3}{*}{1} & DRT & 2.43 & 45.16 & 10.58 \\
\hline & HLT & 0.74 & 24.64 & 12.34 \\
\hline & LRT & 0.64 & 24.40 & 12.29 \\
\hline \multirow{3}{*}{5} & DRT & 6.78 & 57.06 & 12.41 \\
\hline & HLT & 3.39 & 42.96 & 11.72 \\
\hline & LRT & 2.74 & 37.95 & 11.96 \\
\hline \multirow{3}{*}{10} & DRT & 11.37 & 63.38 & 15.30 \\
\hline & HLT & 7.16 & 52.83 & 13.38 \\
\hline & LRT & 6.04 & 46.65 & 13.39 \\
\hline \multicolumn{5}{|c|}{ Scene 2} \\
\hline \multirow{3}{*}{0.5} & $\overline{\text { DRT }}$ & 0.66 & 22.74 & 8.39 \\
\hline & HLT & 0.51 & 16.37 & 8.90 \\
\hline & LRT & 0.49 & 13.67 & 9.15 \\
\hline \multirow{3}{*}{1} & $\overline{\text { DRT }}$ & 1.00 & 27.43 & 8.23 \\
\hline & HLT & 0.97 & 21.20 & 8.82 \\
\hline & LRT & 0.99 & 17.75 & 9.19 \\
\hline \multirow{3}{*}{5} & DRT & 3.75 & 43.05 & 9.03 \\
\hline & HLT & 4.92 & 38.21 & 10.66 \\
\hline & LRT & 6.22 & 34.71 & 12.18 \\
\hline \multirow{3}{*}{10} & DRT & 7.76 & 51.49 & 11.51 \\
\hline & HLT & 10.11 & 49.11 & 14.22 \\
\hline & LRT & 13.56 & 47.52 & 17.49 \\
\hline
\end{tabular}

map obtained by the thresholding CFAR algorithm applied to the DRT, HLT, and LRT statistics, where the significance level of the test $\alpha_{c}$ is chosen equal to $1 \%$. It is worth to notice that with the DRT statistics, more regions are detected than those using the HLT statistics. 


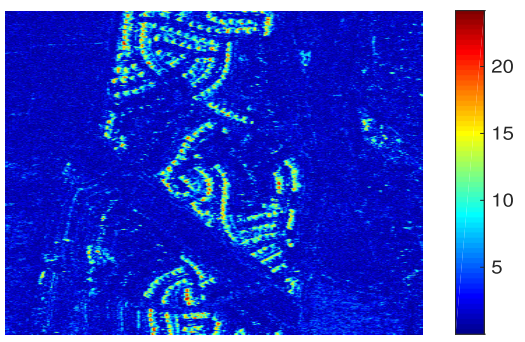

(a1) $\ln \left[\max \left\{\tau_{\mathrm{DRT}}, \tau_{\mathrm{DRT}}^{\prime}\right\}\right]$

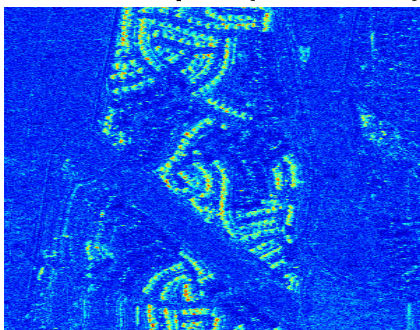

(a1') $\ln \left[\max \left\{\tau_{\mathrm{HLT}}, \tau_{\mathrm{HLT}}^{\prime}\right\}\right]$

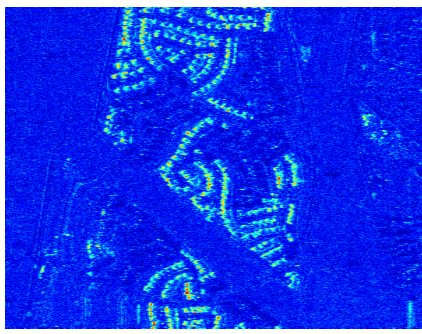

(a1") $\tau_{\text {LRT }}$

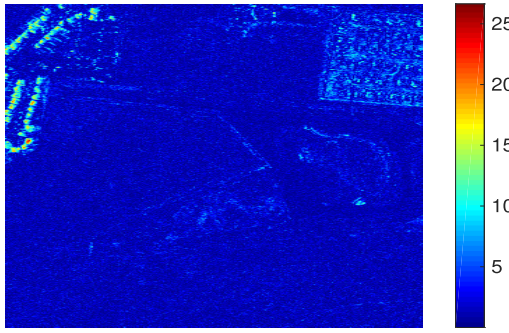

(a2) $\ln \left[\max \left\{\tau_{\mathrm{DRT}}, \tau_{\mathrm{DRT}}^{\prime}\right\}\right]$

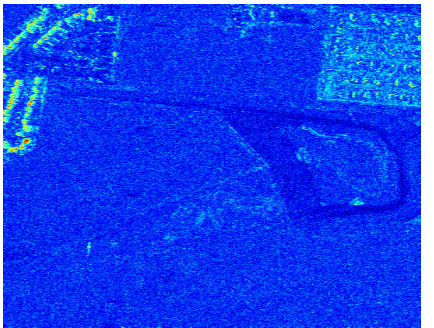

(a2') $\ln \left[\max \left\{\tau_{\mathrm{HLT}}, \tau_{\mathrm{HLT}}^{\prime}\right\}\right]$

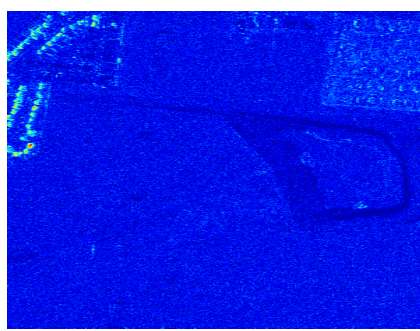

(a2") $\tau_{\mathrm{LRT}}$

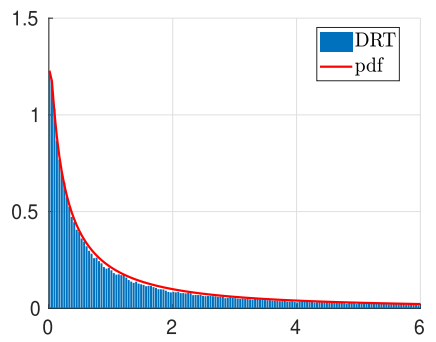

(b1)

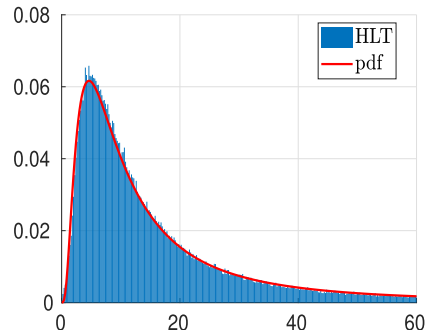

(b1')

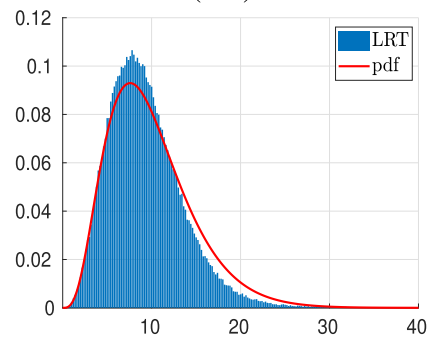

(b1")

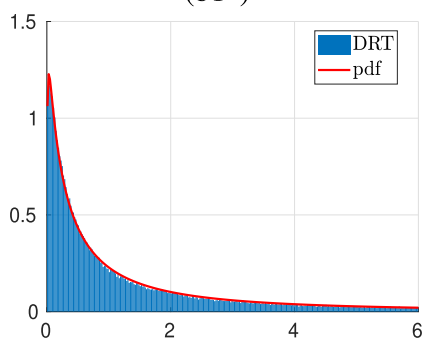

(b2)

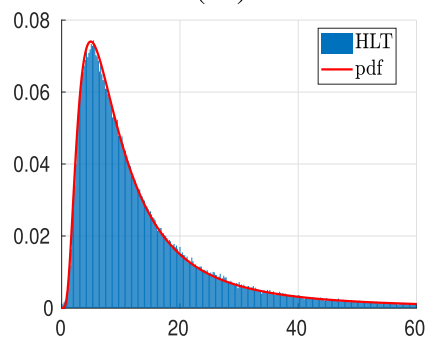

(b2')

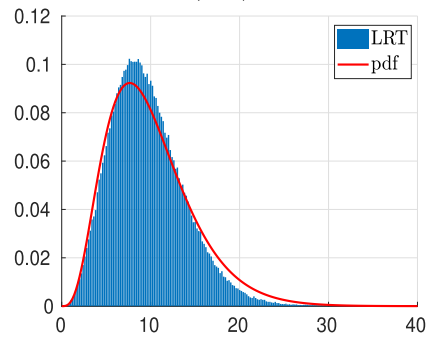

(b2")

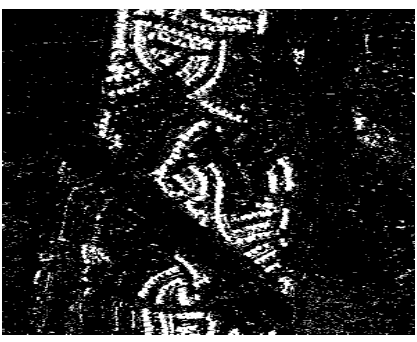

(c1) DRT change detection map

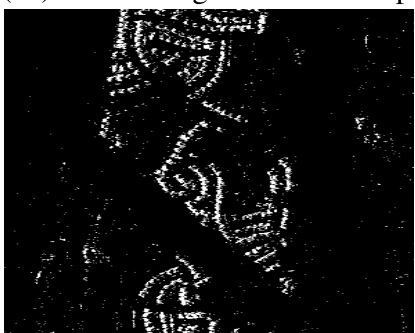

(c1') HLT change detection map

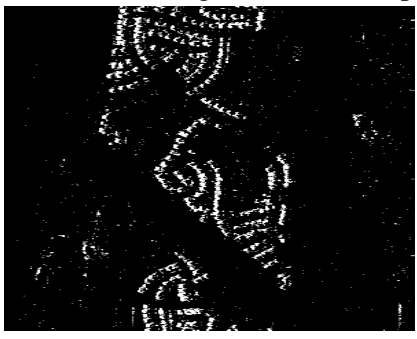

(c1") LRT change detection map

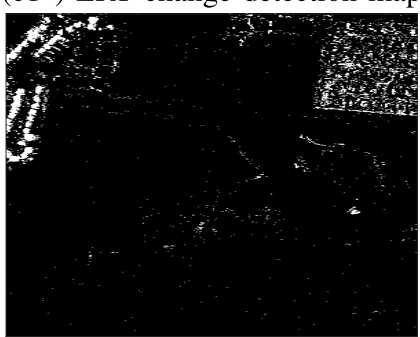

(c2) DRT change detection map

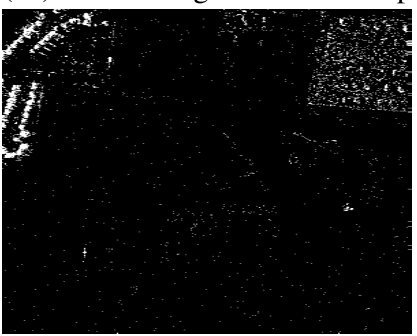

(c2') HLT change detection map

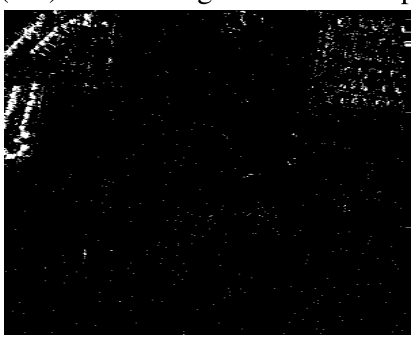

(c2”) LRT change detection map

Fig. 9. Comparison between DRT, HLT, and LRT statistics for real experimental data set over Los Angeles. (ai) Logarithm of max $\left\{\tau_{\mathrm{DRT}}, \tau_{\mathrm{DRT}}^{\prime}\right\}$ for scene i, $\mathrm{i} \in\{1,2\}$. (ai') Logarithm of $\max \left\{\tau_{\mathrm{HLT}}, \tau_{\mathrm{HLT}}^{\prime}\right\}$. (ai') $\tau_{\mathrm{LRT}}$. Plots of normalized histograms and estimated distribution for (bi) DRT, (bi') HLT, and (bi') LRT detector. Change detection map at $1 \%$ significance level obtained from: (ci) DRT, (ci') HLT, and (ci") LRT statistic.

\section{ACCEPTED MANUSCRIPT}



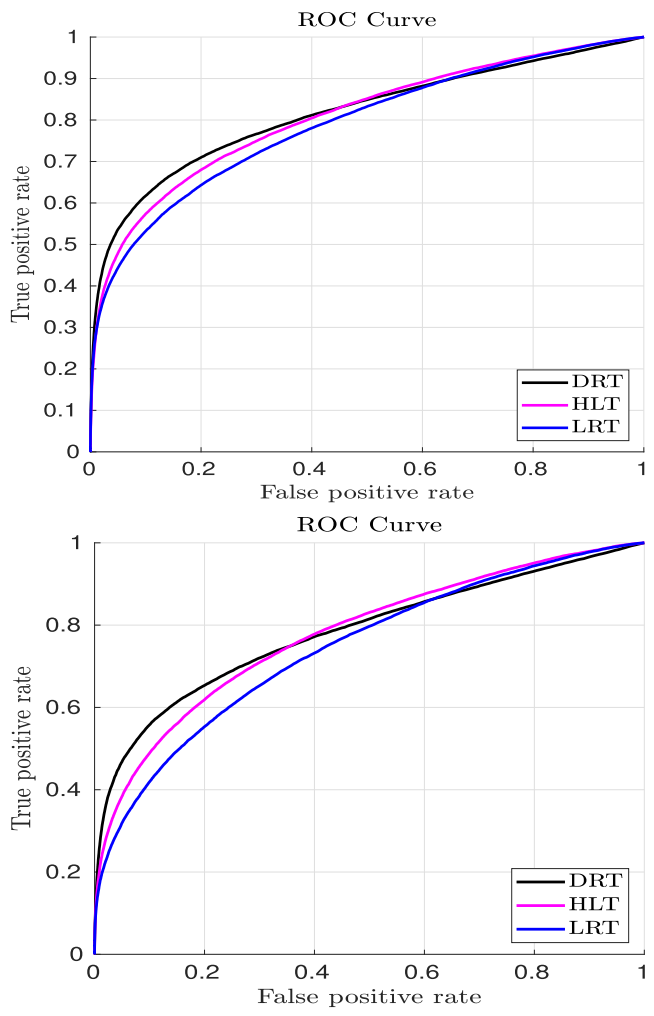

Fig. 10. ROC curves comparison between DRT, HLT, and LRT statistics for real PolSAR data set 2. Top: Scene1. Bottom: Scene2. The DRT detector performs better than the HLT and LRT detectors, especially for a low FPR.

For a final comparison between these change detectors, we draw Table VI. It reports the detection rate, the measured FAR, and the overall error rate computed for the three detectors for scenes 1 and 2, at four different significance levels of the test $\alpha_{c}$. The DRT statistic obtains the highest detection rate in this example, followed by the HLT statistic. The LRT statistic gives the worst performance. The DRT also achieves a lower overall error rate compared to the HLT and LRT statistics except for scene 1 when $\alpha_{c} \geq 5 \%$. Regarding the measured FAR, the DRT gives values close to the specified FAR in most cases (cases of scene 2), but in other cases, the values are far apart (cases of scene 1). The performance of the DRT statistic is evaluated as well through the ROC curve and compared with that of the HLT and LRT statistic. Fig. 10 shows the corresponding ROC curves of the three detectors, indicating a better performance for the DRT statistic for low FPR values followed by the HLT and then by the LRT. The AUC is also provided for this example. For scene 1, it is $81.60 \%$ for the DRT, $80.42 \%$ for the HLT, and $78.96 \%$ for the LRT. For scene 2, it is $78.31 \%$ for the DRT, $77.77 \%$ for the HLT, and $74.41 \%$ for the LRT. Indeed, the AUC given by the DRT statistic is larger than the others.

\section{CONCLUSION}

The DRT statistic has been proposed for change detection in multilook PolSAR images. Under the null hypothesis corresponding to no change, the statistic follows exactly Wilks's lambda distribution of the second kind depending on the ENL and the covariance matrix dimension. The fit ability is evaluated quantitatively using the KS goodness-of-fit test. With this statistic test distribution, the decision threshold can be efficiently determined at a specified probability of false alarm by using the CFAR-threshold method. The performance of the method has been evaluated on simulated and real multilook PolSAR data where ground-truth data were available and has been compared to the performance of the known HLT and LRT detectors. The results in terms of measured FAR, detection rate, ROC curve, and AUC have shown that the proposed DRT statistic outperforms the HLT and LRT performances especially for low ENL values and can perform a binary change detection map very close to the ground-truth data. The method can be extended to a heterogeneous model by considering the texture in the change detection process. The presence of texture can be useful to improve the detection, but additional texture parameters need to be estimated.

\section{APPENDIX A}

The characteristic function of the random variable $V=$ $\ln \left((2 L)^{d}(|\mathbf{X}| /|\boldsymbol{\Sigma}|)\right)$ is given as follows:

$$
\begin{aligned}
\psi_{V}(t) & =E\left\{e^{i t V}\right\}=E\left\{e^{i t \ln \left((2 L)^{d}\left|\frac{\mathbf{X} \mid}{\mid \Sigma}\right|\right)}\right\} \\
& =E\left\{\left((2 L)^{d} \frac{|\mathbf{X}|}{|\boldsymbol{\Sigma}|}\right)^{i t}\right\} \\
& =E\left\{\left(2^{d} \frac{|L \mathbf{X}|}{|\boldsymbol{\Sigma}|}\right)^{i t}\right\} .
\end{aligned}
$$

Let us consider the Hermitian matrix $\mathbf{Z}=L \mathbf{X}$, which follows the nonsingular complex Wishart distribution. The pdf of $\mathbf{Z}$ is as follows:

$$
f_{\mathbf{Z}}(\mathbf{Z})=\frac{|\mathbf{Z}|^{L-d}}{\Gamma_{d}(L)|\boldsymbol{\Sigma}|^{L}} \operatorname{etr}\left(-\Sigma^{-1} \mathbf{Z}\right)
$$

Then, the integral over all the positive definite complex Hermitian matrices verifies

$$
\int_{\boldsymbol{\Omega}_{+}}|\mathbf{Z}|^{L-d} \operatorname{etr}\left(-\mathbf{\Sigma}^{-1} \mathbf{Z}\right) \mathrm{d} \mathbf{Z}=\Gamma_{d}(L)|\mathbf{\Sigma}|^{L} .
$$

We can write that

$$
\int_{\boldsymbol{\Omega}_{+}}|\mathbf{Z}|^{L+i t-d} \operatorname{etr}\left(-\boldsymbol{\Sigma}^{-1} \mathbf{Z}\right) \mathrm{d} \mathbf{Z}=\Gamma_{d}(L+i t)|\mathbf{\Sigma}|^{L+i t} .
$$

As a consequence, (39) can be written as

$$
\begin{aligned}
& \psi_{V}(t) \\
& =\int_{\boldsymbol{\Omega}_{+}}\left(2^{d} \frac{|\mathbf{Z}|}{|\boldsymbol{\Sigma}|}\right)^{i t} f_{\mathbf{Z}}(\mathbf{Z}) \mathrm{d} \mathbf{Z} \\
& =\frac{2^{i t d}}{\Gamma_{d}(L)|\mathbf{\Sigma}|^{L+i t}} \int_{\boldsymbol{\Omega}_{+}}|\mathbf{Z}|^{L+i t-d} \operatorname{etr}\left(-\mathbf{\Sigma}^{-1} \mathbf{Z}\right) \mathrm{d} \mathbf{Z} \\
& =\frac{2^{i t d}}{\Gamma_{d}(L)|\mathbf{\Sigma}|^{L+i t}} \Gamma_{d}(L+i t)|\mathbf{\Sigma}|^{L+i t} \\
& =2^{i t d} \frac{\Gamma_{d}(L+i t)}{\Gamma_{d}(L)} \\
& =2^{i t d} \frac{\Gamma(L+i t) \Gamma(L+i t-1) \ldots \Gamma(L+i t-d+1)}{\Gamma(L) \Gamma(L-1) \ldots \Gamma(L-d+1)} .
\end{aligned}
$$

\section{ACCEPTED MANUSCRIPT}


The pdf of the chi-squared distribution with $2 L$ degrees of freedom $\chi^{2}(2 L)$ is given by

$$
f_{\chi^{2}(2 L)}(x)=\frac{1}{2^{L} \Gamma(L)} x^{L-1} e^{-\frac{x}{2}}
$$

Using the previous pdf, we can write

$$
\int_{0}^{+\infty} x^{L-1} e^{-\frac{x}{2}} \mathrm{~d} x=2^{L} \Gamma(L)
$$

The characteristic function of an $\ln \chi^{2}(2 L)$ random variable is given by

$$
\begin{aligned}
\psi_{\ln \chi^{2}(2 L)}(t) & =E\left\{e^{i t \ln X}\right\}=E\left\{X^{i t}\right\} \\
& =\int_{0}^{+\infty} x^{i t} f_{\chi^{2}(2 L)}(x) \mathrm{d} x \\
& =\frac{1}{2^{L} \Gamma(L)} \int_{0}^{+\infty} x^{L+i t-1} e^{-\frac{x}{2}} \mathrm{~d} x \\
& =2^{i t} \frac{\Gamma(L+i t)}{\Gamma(L)} .
\end{aligned}
$$

The characteristic function of the sum of independent $\ln \chi^{2}(2 L), \ln \chi^{2}(2(L-1)), \ldots, \ln \chi^{2}(2(L-d+1))$ random variables is

$$
\begin{aligned}
& \psi_{\ln \chi^{2}(2 L)+\ln \chi^{2}(2(L-1))+\cdots+\ln \chi^{2}(2(L-d+1))}(t) \\
& =\psi_{\ln \chi^{2}(2 L)}(t) \times \psi_{\ln \chi^{2}(2(L-1))}(t) \times \cdots \times \psi_{\ln \chi^{2}(2(L-d+1))}(t) \\
& =2^{i t d} \frac{\Gamma(L+i t) \Gamma(L+i t-1) \ldots \Gamma(L+i t-d+1)}{\Gamma(L) \Gamma(L-1) \ldots \Gamma(L-d+1)}
\end{aligned}
$$

We can conclude that

$$
\psi_{V}(t)=\psi_{\ln } \chi^{2}(2 L)+\ln \chi^{2}(2(L-1))+\cdots+\ln \chi^{2}(2(L-d+1))(t) .
$$

\section{APPENDIX B}

The Mellin integral transform of the density function $g(z)$ of the product $\prod_{i=0}^{d-1} X_{i}$ of $d$ independent random variables $X_{i}$ with density functions $f_{i}\left(x_{i}\right)$ is given by [33]

$$
\mathcal{M}\{g(z)\}(s)=\prod_{i=0}^{d-1} \mathcal{M}\left\{f_{i}\left(x_{i}\right)\right\}(s) .
$$

Under suitable restrictions [34], [35] satisfied by all density functions, the density function $g(z)$ is given by the following inverse formula:

$$
g(z)=\frac{1}{2 \pi \mathrm{i}} \int_{\mathcal{L}} z^{-s} \prod_{i=0}^{d-1} \mathcal{M}\left\{f_{i}\left(x_{i}\right)\right\}(s) \mathrm{d} s .
$$

Since the Mellin integral transform of $1 /(1+x)^{L_{x}-i+L_{y}-i}$ is given by

$$
\mathcal{M}\left\{\frac{1}{(1+x)^{L_{x}-i+L_{y}-i}}\right\}(s)=\frac{\Gamma\left(L_{x}-i+L_{y}-i-s\right) \Gamma(s)}{\Gamma\left(L_{x}-i+L_{y}-i\right)}
$$

and for any function $f(x)$

$$
\mathcal{M}\left\{x^{L_{x}-i-1} f(x)\right\}(s)=\mathcal{M}\{f(x)\}\left(s+L_{x}-i-1\right)
$$

it follows that the density function of the Beta distribution of the second kind has the following Mellin transform:

$\mathcal{M}\left\{f_{i}\left(x_{i}\right)\right\}(s)=\frac{\Gamma\left(1+L_{y}-i-s\right)}{\Gamma\left(L_{y}-i\right)} \frac{\Gamma\left(L_{x}-i-1+s\right)}{\Gamma\left(L_{x}-i\right)}$.

Then, the Mellin integral transform of the pdf $g(z)$ of the product of $d$ independent random Beta type II variables is

$$
\mathcal{M}\{g(z)\}(s)=\prod_{i=0}^{d-1} \frac{\Gamma\left(1+L_{y}-i-s\right)}{\Gamma\left(L_{y}-i\right)} \frac{\Gamma\left(L_{x}-i-1+s\right)}{\Gamma\left(L_{x}-i\right)} .
$$

Accordingly, the $g(z)$ pdf is written otherwise as a function of the inverse Mellin transform and is given as follows:

$$
\begin{aligned}
& =\frac{1}{2 \pi \mathrm{i}} \int_{\mathcal{L}} z^{-s} \prod_{i=0}^{d-1} \frac{\Gamma\left(1+L_{y}-i-s\right)}{\Gamma\left(L_{y}-i\right)} \frac{\Gamma\left(L_{x}-i-1+s\right)}{\Gamma\left(L_{x}-i\right)} \mathrm{d} s \\
& =\frac{A}{2 \pi \mathrm{i}} \int_{\mathcal{L}} z^{s} \prod_{i=0}^{d-1} \Gamma\left(1+L_{y}-i+s\right) \Gamma\left(L_{x}-i-1-s\right) \mathrm{d} s
\end{aligned}
$$

which is the Meijer G-function multiplied by constant $A$ with $b_{j}=L_{x}-i-1$ and $a_{j}=-\left(L_{y}-i\right)$

$g(z)=A \times G_{d, d}^{d, d}\left(\begin{array}{c}-L_{y},-\left(L_{y}-1\right), \ldots,-\left(L_{y}-(d-1)\right) \\ L_{x}-1, L_{x}-2, \ldots, L_{x}-d\end{array} \mid z\right)$

where $A=\prod_{i=0}^{d-1}\left(1 /\left(\Gamma\left(L_{x}-i\right) \Gamma\left(L_{y}-i\right)\right)\right)$.

The cumulative distribution function $G(t)=\int_{0}^{t} g(z) \mathrm{d} z$ is obtained by integrating (65) with respect to the inside contour integral by using

$$
\int_{0}^{t} z^{s} \mathrm{~d} z=(1+s)^{-1} t^{1+s}=t \frac{\Gamma(1+s)}{\Gamma(2+s)} t^{s} .
$$

Again, using the definition of the Meijer G-function (10), this results in

$$
\begin{aligned}
G(t) & \\
= & \frac{A t}{2 \pi i} \int_{\mathcal{L}} t^{s} \prod_{i=0}^{d-1} \Gamma\left(1+L_{y}-i+s\right) \Gamma\left(L_{x}-i-1-s\right) \\
& \times \frac{\Gamma(1+s)}{\Gamma(2+s)} \mathrm{d} s \\
= & A t \times \\
& G_{d+1, d+1}^{d, d+1}\left(\begin{array}{c}
-L_{y},-\left(L_{y}-1\right), \ldots,-\left(L_{y}-(d-1)\right), 0 \\
L_{x}-1, L_{x}-2, \ldots, L_{x}-d,-1
\end{array} \mid t\right) .
\end{aligned}
$$

The Meijer G-function is viewed as an inverse Mellin transform

$$
G_{p, q}^{m, n}\left(\begin{array}{c}
a_{1}, \ldots, a_{p} \\
b_{1}, \ldots, b_{q}
\end{array} \mid z\right)=\frac{1}{2 \pi i} \int_{\mathcal{L}} z^{-s} \Phi_{Z}(s) \mathrm{d} s
$$

where $\Phi_{Z}(s)=\mathcal{M}\{g(z)\}(s)$ is the Mellin transform of the $g(z)$ pdf. The $k$ th moment $E\left\{Z^{k}\right\}$ is given by

$$
E\left\{Z^{k}\right\}=\left.\Phi_{Z}(s)\right|_{s=k+1}=\prod_{i=0}^{d-1} \frac{\Gamma\left(L_{y}-i-k\right)}{\Gamma\left(L_{y}-i\right)} \frac{\Gamma\left(L_{x}-i+k\right)}{\Gamma\left(L_{x}-i\right)} .
$$

\section{ACCEPTED MANUSCRIPT}




\section{REFERENCES}

[1] L. Gueguen, P. Soille, and M. Pesaresi, "Change detection based on information measure," IEEE Trans. Geosci. Remote Sens., vol. 49, no. 11, pp. 4503-4515, Nov. 2011.

[2] E. Erten, A. Reigber, L. Ferro-Famil, and O. Hellwich, "A new coherent similarity measure for temporal multichannel scene characterization," IEEE Trans. Geosci. Remote Sens., vol. 50, no. 7, pp. 2839-2851, Jul. 2012.

[3] L. Gueguen and M. Datcu, "Mixed information measure: Application to change detection in Earth observation," in Proc. Anal. Multi-Temporal Remote Sens. Images, Mystic, CT, USA, Jul. 2009.

[4] A. D. C. Nascimento, R. J. Cintra, and A. C. Frery, "Hypothesis testing in speckled data with stochastic distances," IEEE Trans. Geosci. Remote Sens., vol. 48, no. 1, pp. 373-385, Jan. 2010.

[5] V. Akbari, S. N. Anfinsen, A. P. Doulgeris, T. Eltoft, G. Moser, and S. B. Serpico, "Polarimetric SAR change detection with the complex Hotelling-Lawley trace statistic," IEEE Trans. Geosci. Remote Sens., vol. 54, no. 7, pp. 3953-3966, Mar. 2016.

[6] E. J. M. Rignot and J. J. van Zyl, "Change detection techniques for ERS-1 SAR data," IEEE Trans. Geosci. Remote Sens., vol. 31, no. 4, pp. 896-906, Jul. 1993.

[7] Y. Bazi, L. Bruzzone, and F. Melgani, "An unsupervised approach based on the generalized Gaussian model to automatic change detection in multitemporal SAR images," IEEE Trans. Geosci. Remote Sens., vol. 43, no. 4, pp. 874-887, Apr. 2005.

[8] B. Hou, Q. Wei, Y. Zheng, and S. Wang, "Unsupervised change detection in SAR image based on gauss-log ratio image fusion and compressed projection," IEEE J. Sel. Topics Appl. Earth Observ. Remote Sens., vol. 7, no. 8, pp. 3297-3317, Aug. 2014.

[9] P. Lombardo and T. M. Pellizzeri, "Maximum likelihood signal processing techniques to detect a step pattern of change in multitemporal SAR images," IEEE Trans. Geosci. Remote Sens., vol. 40, no. 4, pp. 853-870, Apr. 2002.

[10] K. Conradsen, A. A. Nielsen, J. Schou, and H. Skriver, "A test statistic in the complex wishart distribution and its application to change detection in polarimetric SAR data," IEEE Trans. Geosci. Remote Sens., vol. 41, no. 1, pp. 4-19, Jan. 2003.

[11] K. Conradsen, A. A. Nielsen, and H. Skriver, "Change detection in polarimetric SAR data over several time points," in Proc. IEEE Geosci. Remote Sens. Symp., Jul. 2014, pp. 4540-4543.

[12] K. Conradsen, A. A. Nielsen, and H. Skriver, "Determining the points of change in time series of polarimetric SAR data," IEEE Trans. Geosci. Remote Sens., vol. 54, no. 5, pp. 3007-3024, May 2016.

[13] A. A. Nielsen, K. Conradsen, and H. Skriver, "Change detection in full and dual polarization, Single- and multifrequency SAR data," IEEE J. Sel. Topics Appl. Earth Observ. Remote Sens., vol. 8, no. 8, pp. 4041-4048, Aug. 2015.

[14] P. R. Kersten, J. S. Lee, and T. L. Ainsworth, "A comparison of change detection statistics in POLSAR images," in Proc. IEEE Int. Geosci. Remote Sens. Symp., Jul. 2005, pp. 4836-4839.

[15] J. R. Bunch and R. D. Fierro, "A constant-false-alarm-rate algorithm," Linear Algebra Appl., vol. 172, pp. 231-241, Jul. 1992.

[16] S. N. Anfinsen, A. P. Doulgeris, and T. Eltoft, "Estimation of the equivalent number of looks in polarimetric synthetic aperture radar imagery," IEEE Trans. Geosci. Remote Sens., vol. 47, no. 11, pp. 3795-3809, Nov. 2009.

[17] T. Pham-Gia, "Exact distribution of the generalized Wilks's statistic and applications," J. Multivariate Anal., vol. 99, no. 8, pp. 1698-1716, Sep. 2008

[18] N. Otsu, "A threshold selection method from gray-level histograms," IEEE Trans. Syst., Man, Cybern., vol. 9, no. 1, pp. 62-66, Jan. 1979.

[19] J. Kittler and J. Illingworth, "Minimum error thresholding," Pattern Recognit., vol. 19, no. 1, pp. 41-47, Jan. 1986.

[20] M. Ghanbari and V. Akbari, "Unsupervised change detection in polarimetric SAR data with the hotelling-lawley trace statistic and minimumerror thresholding," IEEE J. Sel. Topics Appl. Earth Observ. Remote Sens., vol. 11, no. 12, pp. 4551-4562, Dec. 2018.

[21] J.-S. Lee and E. Pottier, Polarimetric Radar Imaging: From Basics to Application. Boca Raton, FL, USA: CRC Press, 2009.

[22] N. R. Goodman, "Statistical analysis based on a certain multivariate complex Gaussian distribution," Ann. Math. Statist., vol. 34, no. 1, pp. 152-177, Mar. 1963.
[23] A. M. Mathai, Jacobians of Matrix Transformations and Functions of Matrix Argument. Singapore: World Scientific, 1997.

[24] N. R. Goodman, "The distribution of the determinant of a complex Wishart distributed matrix," Ann. Math. Statist., vol. 34, no. 1, pp. 178-180, Mar. 1963.

[25] Y. L. Luke, The Special Functions and Their Approximations/YudellL. Luke. New York, NY, USA: Academic, 1969.

[26] L. Tao, C. Hao-gui, X. Ze-min, and G. Jun, "Texture-invariant estimation of equivalent number of looks based on trace moments in polarimetric radar imagery," IEEE Geosci. Remote Sens. Lett., vol. 11, no. 6, pp. 1129-1133, Jun. 2014

[27] N. Bouhlel, "Parameter estimation of multilook polarimetric SAR data based on fractional determinant moments," IEEE Geosci. Remote Sens. Lett., vol. 16, no. 7, pp. 1075-1079, Jul. 2019.

[28] V. Akbari, "Multitemporal analysis of multipolarization synthetic aperture radar images for robust surface change detection," Ph.D. dissertation, Dept. Phys. Technol., Univ. Tromsø, Troms $\varnothing$, Norway, Jun. 2013.

[29] M. Liu, H. Zhang, C. Wang, and F. Wu, "Change detection of multilook polarimetric SAR images using heterogeneous clutter models," IEEE Trans. Geosci. Remote Sens., vol. 52, no. 12, pp. 7483-7494, Dec. 2014.

[30] N. Bouhlel and S. Meric, "Multilook polarimetric SAR change detection using stochastic distances between matrix-variate Gd0 distributions," IEEE Trans. Geosci. Remote Sens., vol. 58, no. 10, pp. 6823-6843, Oct. 2020.

[31] V. Akbari, S. N. Anfinsen, A. P. Doulgeris, and T. Eltoft, "A change detector for polarimetric SAR data based on the relaxed Wishart distribution," in Proc. IEEE Int. Geosci. Remote Sens. Symp. (IGARSS), Jul. 2015, pp. 3806-3809.

[32] S. Cui, G. Schwarz, and M. Datcu, "A benchmark evaluation of similarity measures for multitemporal SAR image change detection," IEEE J. Sel. Topics Appl. Earth Observ. Remote Sens., vol. 9, no. 3, pp. 1101-1118, Mar. 2016.

[33] M. D. Springer and W. E. Thompson, "The distribution of products of independent random variables," SIAM J. Appl. Math., vol. 14, no. 3, pp. 511-526, Mar. 1966.

[34] E. Titchmarsh, Introduction to the Theory of Fourier Integrals. London, U.K.: Oxford Univ. Press, 1937.

[35] M. D. Springer and W. E. Thompson, "The distribution of products of beta, gamma and Gaussian random variables," SIAM J. Appl. Math., vol. 18 , no. 4, pp. 721-737, Jun. 1970.

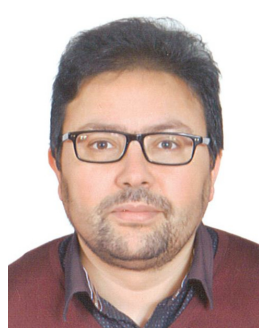

Nizar Bouhlel (Member, IEEE) received the engineering degree in electrical engineering and the master's degree in communication systems from the Ecole Nationale d'Ingénieurs de Tunis (ENIT), Tunisia, in 1997 and 1999, respectively, and the $\mathrm{Ph} . \mathrm{D}$. degree in applied mathematics from the University of Paris Descartes, MAP5, France, in 2006. From 2007 to 2011, he worked as a Researcher at the Faculty of Sciences of Monastir, Communication Department. From 2012 to 2020, he was a PostDoctoral Researcher in several French laboratories: Laboratoire d'Imagerie Biomédicale (LIB), UPMC; Laboratoire des Sciences du Numérique (LS2N), ECN; Institute of Electronics and Telecommunications of Rennes (IETR) with the team SHINE; and INSA Rennes and IETR at CentraleSupélec, Rennes, France. In December 2020, he was hired as an Assistant Professor at Institut Agro, Statistics and Computer Science Department, Angers, France, and at the Research Institute on Horticulture and Seeds (IRHS), Team "Imagerie pour l'Horticulture et le Phénotypage" (ImHorPhen). His research focuses on stochastic signal and image processing, radar signal processing, and signal processing for digital communications. 


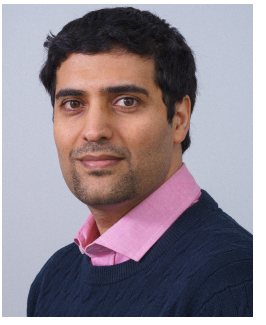

Vahid Akbari (Member, IEEE) received the M.Sc. degree (summa cum laude) in remote sensing from the University of Tehran, Iran, in 2009, and the Ph.D. degree in physics, majoring in earth observation, from the UiT The Arctic University of Norway, Tromsø, Norway, in 2013.

He continued his research in radar remote sensing as a Post-Doctoral Research Fellow with the Department of Physics and Technology, UiT The Arctic University of Norway and Norwegian Instiute of Bioeconomy Research. He has been a Visiting Scientist with the Signal Processing and Telecommunications Laboratory, Department of Electrical, Electronic, Telecommunications Engineering and Naval Architecture, University of Genoa, Italy, in 2011, and a Visiting Researcher with the German Geoscience Center (GFZ), Potsdom, Germany, in 2008. He has been an Assistant Professor of Remote Sensing with the University of Tehran in 2015. He is currently a Research Fellow with the Division of Computing Science and Mathematics, University of Stirling. His research interests include development of methods in machine learning, pattern recognition, and image processing to extract information of land and ocean with radar remote sensing data.

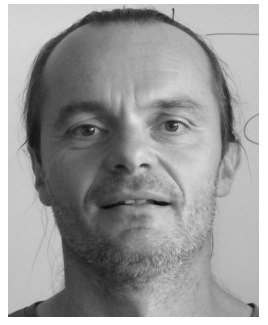

Stéphane Méric (Member, IEEE) received the Electrical Engineer Diploma from the National Institute for the Applied Sciences (INSA), Rennes, France, and the M.S. degree in signal processing and telecommunications from the University of Rennes 1, both in 1991, the Ph.D. degree in electronics from INSA, in 1996, and the HDR (habilitation à diriger des recherches) from the University of Rennes 1 , in 2016 .

Since 2000, he has been an Assistant Professor at INSA. In 2005, he joined the SAPHIR Team (IETR - CNRS UMR 6164, Rennes). He was interested in using SAR data in radargrammetric applications. Furthermore, he is working on radar system (CW, FMCW) dedicated to specific SAR applications (radar imaging in motorway context, remote sensing, MIMO configuration, passive radar imaging) and remote sensing applications. His education activities are about analog electronics, signal processing, radar and radar imaging, as well as electromagnetic diffraction. He is currently the Head Master of the Communication System and Network Department, INSA Rennes. He is a co-author of more than 40 conference papers, 11 journal papers, two book chapters, and one patent. He has been supervising eight Ph.D. students and currently six Ph.D. students.

Dr. Méric was the EuRAD 2019 and the Automotive Forum 2019 TPC CoChair. He has set with the educational team and teaching department in an engineering school at Oujda, Morrocco. 